\title{
Epidemiologische Faktoren bei verschiedenen klinischen Formen der Endometriose Eine Fall-Fall-Untersuchung
}

Frauenklinik des Universitätsklinikums Erlangen

Der Medizinischen Fakultät

der Friedrich-Alexander-Universität

Erlangen-Nürnberg

zur

Erlangung des Doktorgrades Dr. med.

vorgelegt von

Simon Lorenz Blum

aus Bern (Schweiz) 


\section{Als Dissertation genehmigt von der \\ Medizinischen Fakultät der Friedrich-Alexander-Universität Erlangen-Nürnberg}

Vorsitzender des Promotionsorgans: Prof. Dr. Dr. h.c. J. Schüttler

Gutachter:

Gutachter:

Tag der mündlichen Prüfung:
Prof. Dr. Peter Andreas Fasching

Prof. Dr. Matthias Beckmann

27. November 2018 



\section{Inhaltsverzeichnis}

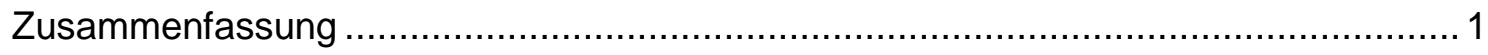

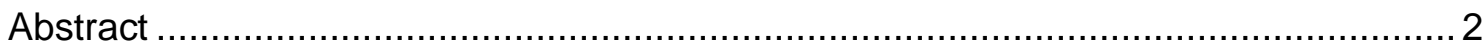

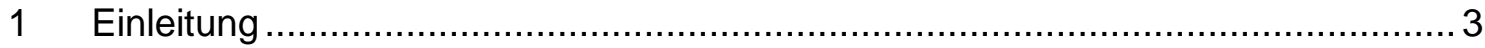

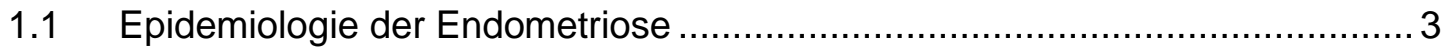

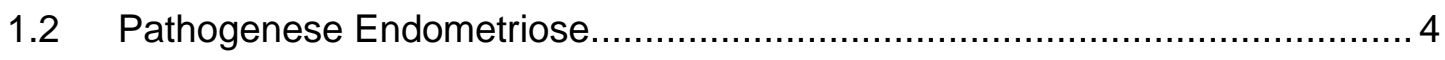

1.3 Patientencharakteristika und Risikofaktoren ........................................ 7

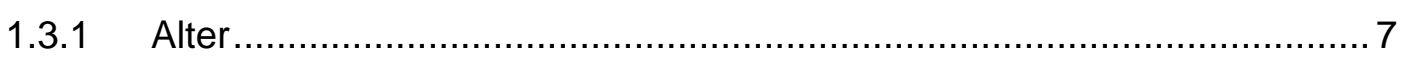

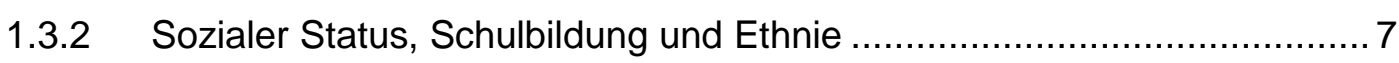

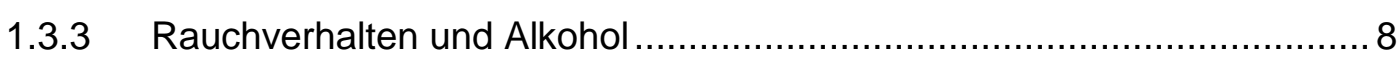

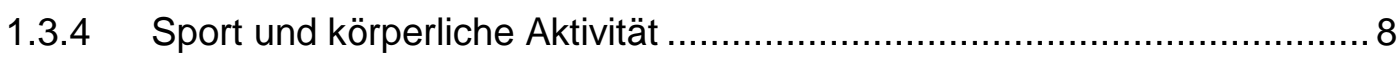

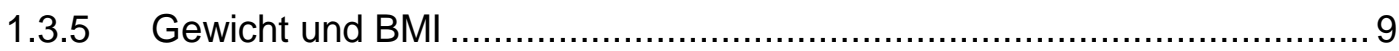

1.3.6 Alter bei Menarche, Zyklusdauer und Blutungslänge ........................... 9

1.3.7 Schwangerschaft und Stillzeit ........................................................... 10

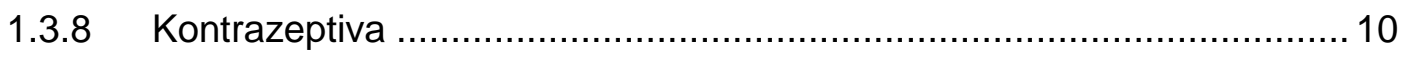

1.3.9 Schmerzen als Risikofaktor ................................................... 11

1.4 Unterschiede von Patientencharakteristika bei verschiedenen Erscheinungsformen der Endometriose ..................................................... 12

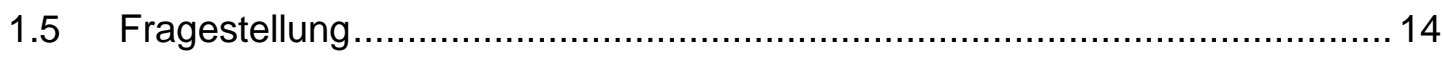

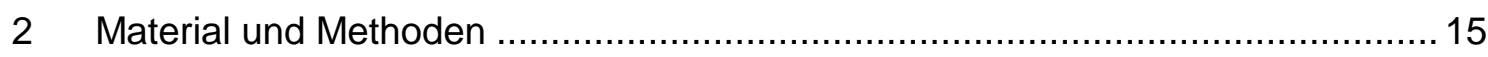

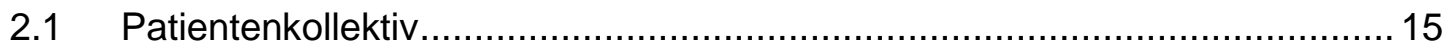

2.2 Populationen und Erstdiagnosefindung ............................................... 17

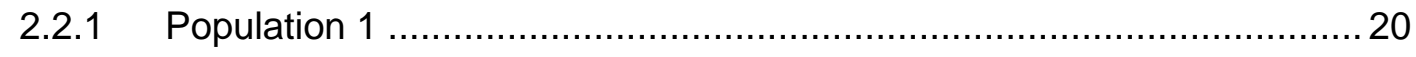

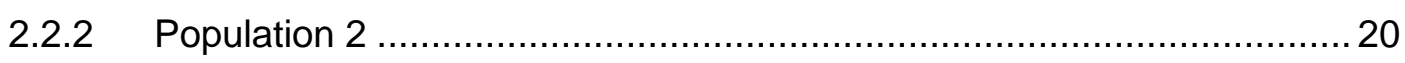

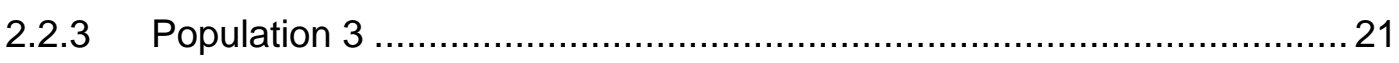

2.2.4 Einteilung der Endometriosepatientinnen in Gruppen ......................... 21

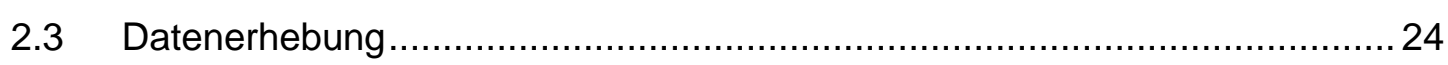

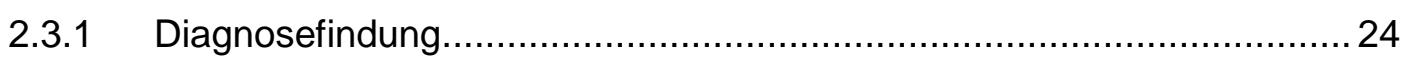

2.3.2 Lokalisation und Art der Endometriose ........................................... 25

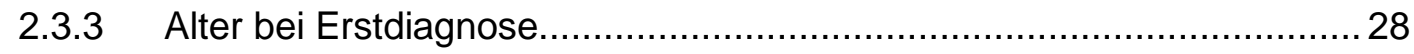

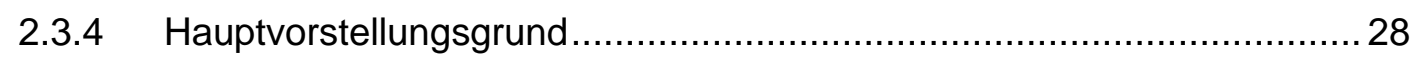

2.3.5 Anzahl Schwangerschaften und Anzahl Lebendgeburten bei Erstvorstellung .................................................................................. 28

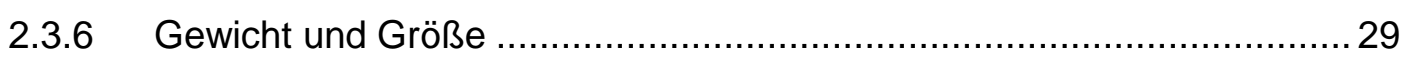




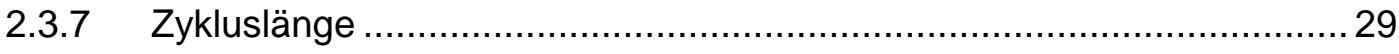

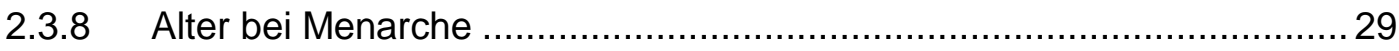

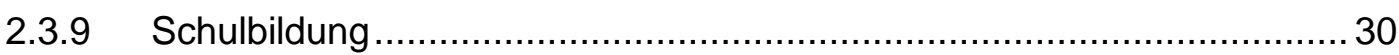

2.4 Dokumentation und Datensicherheit .................................................... 30

2.5 Statistische Auswertung ................................................................ 30

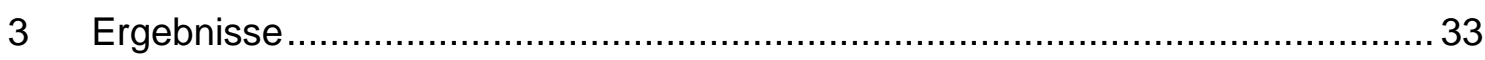

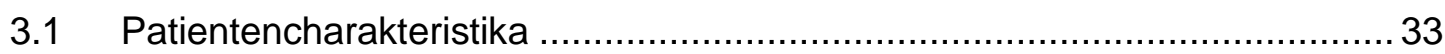

3.2 Einfaktorielle Varianzanalyse (ANOVA) ................................................... 39

3.3 Chi-Quadrat-Test und Kreuztabellen ........................................................ 41

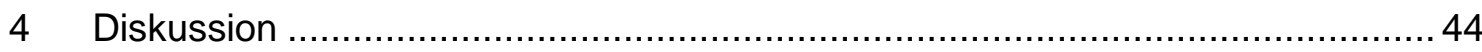

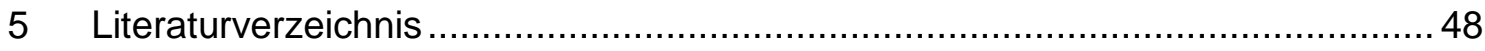

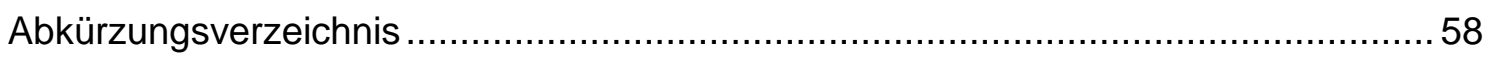

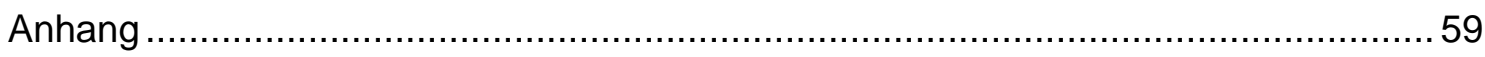

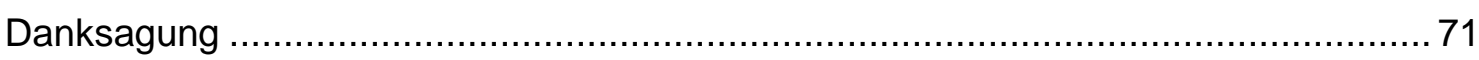




\section{Zusammenfassung}

Hintergrund und Ziele: Bei vielen Erkrankungen kann ein relativ heterogenes Patientenkollektiv in verschiedene Untergruppen unterteilt werden. Für die Endometriose, obwohl eine der häufigsten Erkrankungen der Frau im fertilen Alter, ist eine solche Einteilung bisher nicht bekannt. Ziel dieser Fall-Fall-Studie ist es, verschiedene Patientencharakteristika wie Alter bei Erstdiagnose, BMI, Zykluslänge, Alter bei Menarche, Vorstellungsgrund, Schwangerschaftsraten, Lebendgeburtenraten und Schulbildung in Untergruppen zu untersuchen.

Methoden: Für die vorliegende retrospektive Fall-Fall-Untersuchung wurden im Zeitraum von Februar 2014 bis Juli 2017 Daten von 1576 Patientinnen im Rahmen der klinischen Tätigkeit mittels eines web-basierten Dokumentationssystems erfasst. Mittels einfaktorieller Varianzanalyse (ANOVA) und Chi-Quadrat-Test wurden fünf verschiedene Patientengruppen auf Unterschiede hinsichtlich der Patientencharakteristika untersucht.

Ergebnisse und Beobachtungen: Das mittlere Alter bei Erstdiagnose lag bei 33,86 Jahren. Es zeigte sich ein signifikanter Unterschied beim mittleren Erstdiagnosealter zwischen den Gruppen. Die Gruppe mit alleiniger Adenomyose wies hierbei das höchste mittlere Alter auf. Weiter wiesen die Daten auf einen Zusammenhang zwischen der Schwangerschaftsrate beziehungsweise Lebendgeburtenrate und den fünf Patientengruppen hin.

Schlussfolgerung: Diese Studie ist ein erster Hinweis darauf, dass die heterogenen Erscheinungsformen der Endometriose nicht als eine einheitliche Erkrankung betrachtet werden sollten. Es sind jedoch noch weitere Studien nötig, um eine klare Einteilung in Subpopulationen vornehmen zu können. 


\begin{abstract}
Background and aims: In many diseases, a heterogeneous collective can be divided into subgroups. For the endometriosis, such a categorization is not known, although it is one of the most common diseases in reproductive age of women. The aim of this study is to investigate patient characteristics such as age at diagnosis, BMI, cycle length, age at menarche, reason for consultation, pregnancy rates, live birth rates and education in subgroups.
\end{abstract}

Methods: For this case-case study, data from 1076 patients were collected from June 2013 to July 2017 using a web-based documentation system. By means of a single factor variance analysis (ANOVA) and Chi-squared test, five groups were examined for differences in patient characteristics.

Results and observations: The mean age at first diagnosis was 33,86 years. There was a significant difference in the mean age between the five groups. The group in which the patients were only diagnosed with adenomyosis showed the highest mean age. Furthermore, the data indicate a link between the pregnancy rate or live birth rate and the groups.

Conclusions: This study is a first indication that the heterogeneous manifestations of endometriosis could be considered as a heterogeneous disease. However, further studies are needed to make a clear classification in subpopulations. 


\section{Einleitung}

\subsection{Epidemiologie der Endometriose}

Unter Endometriose wird eine Ablagerung von endometriumartigen Zellverbänden außerhalb des Cavums Uteri bezeichnet. Es handelt sich dabei um eine zum Teil infiltrativ wachsende benigne Erkrankung (Ulrich et al., 2013). Eine genaue Angabe bezüglich der Prävalenz und Inzidenz der von Endometriose betroffenen Frauen ist mit der aktuellen Studienlage nicht möglich. Die Hauptproblematik liegt hierbei bei einer zur Diagnosestellung notwendigen chirurgischen Intervention. Je nach untersuchtem Patientenkollektiv variieren die Angaben zu Prävalenz sehr stark. Sie reichen von $4 \%$ bis $30 \%$ (Abbas et al., 2012; Mahmood und Templeton, 1991; Moen und Schei, 1997; Nezhat et al., 2008; Signorello et al., 1997; Vigano et al., 2004).

In einer Übersichtsarbeit über 27 Studien variierte die Prävalenz bei Patientinnen mit chronischen Unterbauchschmerzen (chronic pelvic pains, CPPs) von 2,2\% (Levitan et al., 1985) bis $81,7 \%$ (Cornillie et al., 1990). Im Durchschnitt ergab sich eine Prävalenz bei Frauen mit chronischen Unterbauchschmerzen von $28,7 \%$. Bei der Untersuchung zeigte sich eine zunehmende Prävalenz je jünger das Publikationsjahr der Studie war, was mit einer Zunahme der Bekanntheit der Endometriose als Erkrankung zusammenhängen könnte (Guo und Wang, 2006). 


\subsection{Pathogenese Endometriose}

1860 wurde die Endometriose erstmals als eigene Entität von Carl Freiherr von Rokitansky beschrieben (Rokitansky, 1860).

Anfang des 20. Jahrhunderts erkannte Thomas Stephen Cullen, dass es sich bei den Ablagerungen um eine Invasion endometrialen Gewebes handelt. $\mathrm{Er}$ definierte so erstmals einen möglichen Pathomechanismus, durch welchen die endometriale Mucosa das darunterliegende Gewebe infiltriert (Cullen, 1908, 1920). Bereits kurz darauf entwickelte John A. Sampson die Transplantationstheorie. Er ging davon aus, dass durch eine retrograde Menstruationsblutung über die Tuben Endometriumzellen in den Bauchraum gelangen und so die Endometriose-Läsionen verursachen (Sampson, 1921, 1927a). Ein weiterer möglicher Mechanismus zur Verschleppung von Endometriumzellen wurde durch Sampson als Metastasierung beziehungsweise Embolisierung über die venöse Zirkulation des Uterus während einer Menstruationsblutung beschrieben (Sampson, 1927b).

Jedoch kommt eine retrograde Blutung bei $70 \%$ bis $90 \%$ aller prämenopausalen Frauen vor und stellt somit eher den Normalzustand als eine Pathologie dar, was die retrograde Blutung als alleinige Ursache für die Entstehung einer Endometriose unwahrscheinlich macht (Blumenkrantz et al., 1981). Es wird daher davon ausgegangen, dass der Entstehung der Endometriose weitere Prozesse zugrunde liegen (D'Hooghe und Debrock, 2002). Es wird einerseits angenommen, dass sowohl eine kurze Zyklusdauer (Cramer und Missmer, 2002), wie auch eine Obstruktion beim Abfluss der Menstruationsblutung zu einer erhöhten Inzidenz von Endometriose führt (Cramer und Missmer, 2002; Pinsonneault und Goldstein, 1985). Aber auch, dass Entzündungsvorgänge und verschiedene inflammatorische Faktoren zur Adhäsion von Endometriumzellen am Peritoneum führen (D'Hooghe und Debrock, 2002). Untersuchungen zeigten, dass womöglich auch immunologische Einflüsse bei der Implantation von Endometriumzellen entscheidend sind (Dmowski et al., 1981; Oosterlynck et al., 1991). 
Die zweite, weit verbreitete Theorie, die Theorie der Metaplasie, geht davon aus, dass sich Gewebe unter bestimmten Umständen in ein anderes Gewebe mit Übernahme der neuen Gewebefunktionen differenzieren kann. Als mögliche Auslöser hierfür wurden sowohl hormonelle, wie auch entzündliche Einflüsse diskutiert (Meyer, 1919). So ist es möglich, dass während der Embryogenese verschleppte Zellen des müllerschen Ganges durch bislang unbekannte Einflüsse zu einer Differenzierung in Endometriumzellen angeregt werden können (Gruenwald, 1942; Meyer, 1919).

Beide Theorien, die Implantationstheorie, wie die Metaplasietheorie können jedoch nicht gänzlich die Entstehung der Endometriose erklären. Zudem ist es mit den beiden Theorien nicht möglich, die verschiedenen Manifestationsformen und Ausprägungsgrade der Endometriose zu erklären. Es ist daher anzunehmen, dass die Pathogenese weitaus komplizierter abläuft, als es diese beiden Theorien vermuten lassen. Neuere Studien untersuchen daher insbesondere hormonelle und genetische Einflüsse.

Es wird davon ausgegangen, dass es sich bei der Endometriose um eine östrogenabhängige Erkrankung handelt (Takayama et al., 1998; Ulrich et al., 2013; Zanetta et al., 2000). Bislang ist allerdings nicht bekannt, wie und unter welchen Umständen es unter einer Stimulation von Östrogen zu einem Wachstum der Endometrioseherde kommt (Ulrich et al., 2013). Neben zusätzlichen Risikofaktoren wie Umwelteinflüssen, auf die später noch eingegangen wird, werden genetische Einflussfaktoren vermutet (Giudice, 2003; Hadfield et al., 1997; Kennedy et al., 1995; Moen, 1994; Simpson und Bischoff, 2003; Simpson und Bischoff, 2002; Simpson et al., 1980; Stefansson et al., 2002). Hier stehen insbesondere Genabschnitte im Verdacht, die an der östrogenabhängigen hormonellen Regulation beteiligt sind (Renner et al., 2006). Neben einer Überexpression von Östrogen stehen noch andere Zellmechanismen wie vermehrte Expression von Prostaglandinen im Verdacht, das Wachstum von Endometrioseherden zu fördern (Ebert et al., 2005). Vermutet wird ebenfalls ein Ungleichgewicht zwischen der Progesteron- und Östrogenproduktion (Lessey et al., 1992). 
In kürzlich durchgeführten Studien konnte gezeigt werden, dass Endometriose mit einem erhöhten Risiko für Endometriumkarzinom (Yu et al., 2015) und klarzelliges Ovarialkarzinom (Burghaus et al., 2017; Lu et al., 2015; Pearce et al., 2012) assoziiert ist. Dies legt den Schluss nahe, dass beiden Erkrankungen, Endometriose und Ovarialkarzinom ein ähnlicher Pathomechanismus zugrunde liegen könnte (Burghaus et al., 2017).

Trotz vielseitiger Untersuchung konnte bislang der Pathomechanismus zur Entstehung von Endometriose nicht entschlüsselt werden. Hier sind dringend weitere Studien notwendig. 


\subsection{Patientencharakteristika und Risikofaktoren}

\subsubsection{Alter}

Verschiedene Studien zeigten einen Zusammenhang zwischen Alter und einer intraoperativ diagnostizierten Endometriose (Gruppo-Italiano, 1994).

Es kann davon ausgegangen werden, dass das Risiko, an einer Endometriose zu erkranken, nur während des gebärfähigen Alters besteht. Zur Inzidenzrate gibt es jedoch unterschiedliche Daten. Frühere Untersuchungen legten nahe, dass das Risiko, an einer Endometriose zu erkranken, bis zum 44. Lebensjahr zunimmt. Das höchste Risiko, an einer Endometriose zu erkranken, lag zwischen 40 und 44 Jahren. Eine weitere Erkrankungsspitze zeigte sich zwischen 25 und 29 Jahren. Nach dem 44. Lebensjahr nahm das Risiko rasch wieder ab. Neuere Studien gaben das höchste Risiko für die Entstehung einer Endometriose im jüngeren Alter bei 25-29 Jahren an. Hier zeigte sich ebenfalls eine rasche Abnahme des Risikos nach dem 44. Lebensjahr (Houston et al., 1988; Missmer et al., 2004b; Sangi-Haghpeykar und Poindexter, 1995).

\subsubsection{Sozialer Status, Schulbildung und Ethnie}

Es konnte ein Zusammenhang zwischen sozialen Status und der Häufigkeit von Endometriose festgestellt werden. (Missmer et al., 2004b; Neme et al., 2002; Signorello et al., 1997). So steigt das Risiko für Endometriose mit der Zugehörigkeit zu einer Bevölkerungsgruppe mit einem höheren sozialen oder ökonomischen Status (Neme et al., 2002). Es wird allerdings angenommen, dass der Effekt ethnischer Zugehörigkeit und schulischer Bildung eher durch einen damit assoziierten höheren sozialen Status und eine vermehrte Inanspruchnahme medizinischer Leistungen zustande kommt. Hier sind insbesondere kostenaufwändige Infertilitätsbehandlungen zu berücksichtigen (Signorello et al., 1997).

Andere Studien zeigten keinen Zusammenhang zwischen der Schulbildung der Probanden und Endometriose (Saha et al., 2017). 


\subsubsection{Rauchverhalten und Alkohol}

In einigen Studien konnte ein abnehmendes Risiko durch Tabakkonsum nachgewiesen werden. Je früher eine Probandin mit dem Rauchen begonnen hat, desto niedriger war das Risiko an einer Endometriose zu erkranken (Cramer et al., 1986; Eskenazi und Warner, 1997; Missmer et al., 2004b). Jedoch gibt es hierzu widersprüchliche Daten (Gruppo-Italiano, 1999; Heilier et al., 2007), wonach Rauchen in einigen Studien sogar die Entstehung einer Endometriose begünstigt (Saha et al., 2017).

Ein ähnliches Ergebnis zeigt sich beim Alkoholkonsum.

Hier zeigte sich ein erniedrigtes Risiko durch den Konsum von Alkohol (Missmer et al., 2004b). Eine andere Studie konnte einen Zusammenhang zwischen Alkoholkonsum und tiefinfiltrierender Endometriose, nicht aber peritonealer Endometriose nachweisen (Heilier et al., 2007). Einige Studien konnten wiederum keinen signifikanten Zusammenhang zwischen Endometriose und Alkoholkonsum feststellen (Signorello et al., 1997).

\subsubsection{Sport und körperliche Aktivität}

In einigen Studien konnte ein Zusammenhang zwischen körperlicher Aktivität und der Inzidenz von Endometriose festgestellt werden. So wirkt sich körperliche Aktivität und Sport protektiv aus (Cramer et al., 1986; Signorello et al., 1997). Eine Studie konnte sogar nur einen Zusammenhang zwischen moderater körperlicher Aktivität in der Arbeit, nicht aber körperlicher Aktivität in der Freizeit und Endometriose feststellen (Heilier et al., 2007).

Eine neuere Studie wiederum wies keinen signifikanten Zusammenhang zwischen körperlicher Aktivität und Endometriose nach (Vitonis et al., 2010). 


\subsubsection{Gewicht und BMI}

Mit zunehmendem Gewicht oder mit dem aus Körpergröße und Gewicht berechneten Body Mass Index (BMI), steigt das Risiko, an Endometriose zu erkranken (Missmer et al., 2004b; Signorello et al., 1997).

Eine eindeutige Datenlage liegt hierbei jedoch nicht vor. So gibt es eine Studie, die einen Zusammenhang zwischen einem schlanken Körperbau in der Kindheit, zur Zeit der Menarche, sowie zwischen 20-25 Jahren und einem erhöhten Risiko, an Endometriose zu erkranken, zeigte. In derselben Studie konnte sogar ein Zusammenhang zwischen Körpergröße und einem erhöhten Risiko für Endometriose festgestellt werden (Farland et al., 2017b).

In einer kürzlich durchgeführten Metaanalyse von 11 Studien konnte ein niedrigeres Risiko für die Entstehung einer Endometriose mit zunehmendem Body Mass Index nachgewiesen werden (Liu und Zhang, 2017).

\subsubsection{Alter bei Menarche, Zyklusdauer und Blutungslänge}

In einigen Studien konnte ein starker Zusammenhang zwischen einer frühen Menarche und dem Risiko für das Entstehen einer Endometriose festgestellt werden (Missmer et al., 2004a; Saha et al., 2017; Treloar et al., 2010).

Bei anderen Studien konnte hingegen kein statistisch signifikanter Zusammenhang zwischen früher Menarche und Risiko für die Entstehung einer Endometriose nachgewiesen werden (Cramer et al., 1986; Signorello et al., 1997).

Ebenfalls liegen Daten für den Zusammenhang zwischen einem kurzen Menstruationszyklus und dem erhöhten Risiko für Endometriose vor (Burghaus et al., 2011; Cramer et al., 1986; Missmer et al., 2004a; Treloar et al., 2010).

Auch bei der Dauer der Menstruationsblutung konnte ein Zusammenhang mit dem Risiko für die Entstehung einer Endometriose festgestellt werden (Burghaus et al., 2011; Cramer et al., 1986).

Ein Zusammenhang zwischen einer verstärkten Menstruationsblutung und Endometriose ließ sich jedoch nicht feststellen (Treloar et al., 2010). 


\subsubsection{Schwangerschaft und Stillzeit}

Schwangerschaften wirken sich protektiv aus. So sinkt mit jeder Schwangerschaft das Risiko, an Endometriose zu erkranken. Hierbei ist es nicht von Relevanz, in welchem Alter die erste Schwangerschaft eingetreten ist. Insbesondere Nullipara haben demnach das höchste Risiko an Endometriose zu erkranken (Candiani et al., 1991; Gruppo-Italiano, 1999; Missmer et al., 2004a; Parazzini et al., 1995; Saha et al., 2017). Die Anzahl an Lebendgeburten kann allerdings nicht als Prädiktor herangezogen werden (Burghaus et al., 2011).

Es konnte gezeigt werden, dass Patientinnen mit einer Endometriose ein erhöhtes Risiko für Komplikationen in der Frühschwangerschaft, wie Abort und ektope Schwangerschaften haben. Weiter wurden hier vermehrt placenta praevia, vaginale Blutungen in der Schwangerschaft und postpartale Blutungen, wie auch Frühgeburtlichkeit nach 24 Schwangerschaftswochen beobachtet (Burghaus et al., 2011; Saraswat et al., 2017).

Stillen wirkt sich ebenfalls protektiv auf die Entstehung von Endometriose aus. So konnte nachgewiesen werden, dass mit der Dauer des Stillens das Risiko für eine Endometriose abnimmt. Dieser Effekt war insbesondere in den ersten fünf Jahren nach der Geburt am größten. Ein protektiver Effekt durch eine postpartale Amenorrhö konnte hierbei nicht ausgeschlossen werden (Farland et al., 2017a; Missmer et al., 2004a).

\subsubsection{Kontrazeptiva}

Die Einnahme von oralen Kontrazeptiva ist eine etablierte Therapieoption bei Patientinnen, die an Endometriose erkrankt sind (Ulrich et al., 2013; Valle und Sciarra, 2003). Eine therapeutische Einnahme von Kontrazeptiva ist in dieser Situation vor allem im Langzyklus sinnvoll (Vercellini et al., 2003).

Ob orale Kontrazeptiva allerdings auch einen protektiven Effekt auf die Entstehung einer Endometriose haben, ist nicht endgültig geklärt. 
Einige Studien zeigen einen protektiven Effekt durch die Einnahme von oralen Kontrazeptiva. Dieser Effekt war umso größer, je länger die Probandinnen die Kontrazeptiva einnahmen (Buttram, 1979; Kirshon und Poindexter, 1988; Mahmood und Templeton, 1991). In einer Studie wurde ein niedrigeres Risiko durch die Einnahme von oralen Kontrazeptiva beschrieben. Dieser Effekt war allerdings nur dann nachweisbar, wenn die Kontrazeptiva kurzfristig eingenommen wurden, was auf einen nur kurzfristig protektiven Effekt durch die Einnahme von Kontrazeptiva hinweisen könnte (Sangi-Haghpeykar und Poindexter, 1995).

\subsubsection{Schmerzen als Risikofaktor}

Schmerz ist ein sehr subjektives Erlebnis, daher kann anhand der Schmerzintensität nicht auf die Ausdehnung der peritonealen Endometrioseherde geschlossen werden (Koninckx et al., 1991).

Schmerz ist demnach nur ein schlechter Prädiktor, um die Ausdehnung einer Endometriose bei einer Patientin vorherzusagen. In einer Studie konnte allerdings gezeigt werden, dass bei ausgeprägter präoperativer Schmerzsymptomatik das Risiko für ein Rezidiv in den ersten 4 Jahren erhöht ist (Renner et al., 2010). 


\subsection{Unterschiede von Patientencharakteristika bei verschiedenen Erscheinungsformen der Endometriose}

Wie bereits oben beschrieben gibt es eine Vielzahl von Studien, die Patientencharakteristika und Risikofaktoren für Endometriose untersuchen. Jedoch wird hier in der Regel nicht zwischen verschiedenen Erscheinungsformen der Endometriose unterschieden. So gibt es einzelne Untersuchungen, die sich vermehrt mit tiefinfiltrierender Endometriose oder mit Adenomyose befassen, es gibt aber kaum Studien, die verschiedene Erscheinungsformen der Endometriose miteinander vergleichen. Daher gibt es kaum Daten, die Aufschluss darüber geben, ob es sich bei den von uns untersuchten Phänotypen, wie der peritoneale Endometriose, der tiefinfiltrierenden Endometriose, der Adenomyose und der Endometriome tatsächlich um zu unterscheidende Erscheinungsformen oder gar um eigene Erkrankungen handelt.

Eine solche Einteilung wurde versucht, anhand der Morphologie verschiedener Endometriose-Läsionen vorzunehmen (Koninckx und Martin, 1992; Nisolle und Donnez, 1997; Vercellini et al., 2004). Diese haben sich allerdings nicht durchgesetzt.

Handelt es sich bei den unterschiedlichen Formen der Endometriose tatsächlich um unterschiedliche Typen, müssten sich auch unterschiedliche Patientencharakteristika oder Risikoprofile finden lassen. Eine solche Einteilung wäre dann auch für die weitere Erforschung der Endometriose relevant.

In einer Studie wurden Patientencharakteristika bei Patientinnen mit Adenomyose und Endometriose miteinander verglichen. Frauen mit Endometriose waren im Durchschnitt jünger als solche mit Adenomyose. Endometriose war in dieser Studie mit einer positiven Familienanamnese für Endometriose (Mutter oder Schwester) sowie mit Nulliparität assoziiert. Die Adenomyose war hingegen mit einer erhöhten Schwangerschaftsrate, einer frühen Menarche ( $\leq 10$ Jahre), einem kurzen Menstruationszyklus ( $\leq 24$ Tage) und Übergewicht (Templeman et al., 2008) verbunden. 
In anderen Studien wurden Patientinnen mit tiefinfiltrierender Endometriose mit Patientinnen mit peritonealer oder ovarieller Endometriose verglichen. Hier zeigten sich keine Unterschiede beim Alter der Patientinnen (Koninckx et al., 1991; Parazzini et al., 2008). Zudem konnte ein niedrigeres Risiko für die Probandinnen mit einem erhöhten Body Mass Index und mit der steigenden Anzahl der Geburten für alle Gruppen nachgewiesen werden. Die Phänotypen unterschieden sich nicht im Risikoprofil (Parazzini et al., 2008).

Es zeigte sich, dass die Anwesenheit von Endometriomen, aber auch die Tiefe der Infiltration bei tiefinfiltrierender Endometriose mit Schmerzen assoziiert sind (Cornillie et al., 1990; Koninckx et al., 1991). Während Infertilität bei Patientinnen mit peritonealer Endometriose, nicht aber bei tiefinfiltrierender Endometriose auftrat (Cornillie et al., 1990).

Es wird seit langem angenommen, dass Endometriose und insbesondere auch die Adenomyose mit Schmerzen und Dysmenorrhoe zusammenhängt (Bird et al., 1972; Vavilis et al., 1997). Eine neuere Studie konnte jedoch keinen Zusammenhang zwischen Schmerzen und Adenomyose feststellen. Schmerz ist demnach nicht geeignet als Prädiktor für Adenomyose. In derselben Studie konnte ebenfalls gezeigt werden, dass kein Zusammenhang zwischen Blutungsstörungen und Adenomyose bestehen. Es ist daher nicht sicher auszuschließen, dass es sich bei der Adenomyose eher um einen Normalzustand als um eine Erkrankung handelt (Weiss et al., 2009).

In einer Studie konnte nachgewiesen werden, dass durch die Einnahme von oralen Kontrazeptiva vermehrt Endometriome auftraten (Parazzini et al., 1989). Untersuchungen zeigen, dass präoperativ zwar anhand der Schmerzen eine Lokalisation der Endometriose-Läsionen möglich ist (Renner et al., 2012), dies jedoch keinen Aufschluss über die Art der Endometriose gibt. 


\section{$1.5 \quad$ Fragestellung}

Bei vielen Erkrankungen kann ein relativ heterogenes Patientenkollektiv in verschiedene Untergruppen unterteilt werden, um eine mögliche Behandlung individualisierter und effektiver durchzuführen. So kann z.B. beim Mammakarzinom in Abhängigkeit der vom Tumor exprimierten Rezeptoren eine Einteilung vorgenommen werden. Das Patientenkollektiv dieser Untergruppen unterscheidet sich in den Patientencharakteristika wie Alter, Prognose, usw. .

Für die Endometriose, obwohl eine sehr heterogene Erkrankung, ist eine solche Einteilung nicht bekannt.

Ziel dieser Arbeit ist es, verschiedene Patientencharakteristika anhand fünf definierter Patientengruppen zu untersuchen.

\section{Hauptfragestellung}

Gibt es einen Zusammenhang zwischen dem Alter bei Erstdiagnose und den fünf Patientengruppen?

\section{Nebenfragestellung}

Gibt es weitere Patientencharakteristika, die einen Zusammenhang mit den Patientengruppen aufweisen? Untersucht werden sollen hierbei der Body Mass Index, Zykluslänge bei Erstvorstellung, Alter bei Menarche, Hauptvorstellungsgrund bei Erstvorstellung, Anzahl Schwangerschaften bei Erstvorstellung, Anzahl Lebendgeburten bei Erstvorstellung und Schulbildung bei Erstvorstellung. 


\section{Material und Methoden}

\subsection{Patientenkollektiv}

Seit Juni 2013 werden an der Frauenklinik des Universitätsklinikums Erlangen mittels eines web-basierten Dokumentationssystems sämtliche operativ behandelten Patientinnen mit der Diagnose einer Endometriose erfasst (Burghaus et al., 2016). Dies geschieht in Kooperation mit mittlerweile 10 weiteren Endometriosezentren, die mithilfe des web-basierten Dokumentationssystems Daten für die jährliche Zertifizierung als Endometriosezentrum durch die die Stiftung Endometriose-Forschung (SEF), die Europäische Endometriose Liga und die Endometriose Vereinigung Deutschland erfassen.

Seit Beginn der Dokumentation am 18.02.2014 wurden in der Frauenklinik des Universitätsklinikums Erlangen bis zum 04.07.2017 insgesamt 1576 Patientinnen, mit Erstvisiten bis Juli 2011 reichend, dokumentiert. Von diesen Patientinnen wurden 356 Patientinnen für diese Arbeit ausgeschlossen, bei denen kein für die Auswertung adäquates Erstdiagnosedatum bestimmt werden konnte. Von den verbleibenden 1220 Patientinnen mit einer operativen Erstdiagnose wurden weitere 144 Patientinnen ausgeschlossen, bei denen eine eindeutige Einteilung in 16 mögliche Untergruppen bezüglich Art und Lokalisation der Endometriose nicht möglich war. Für die Auswertung ergab sich so ein Kollektiv von 1076 Patientinnen mit einer operativen Erstdiagnose einer Endometriose ohne vorangegangene anamnestische Diagnose einer Endometriose. 


\section{Abbildung 1: Patientenkollektiv}

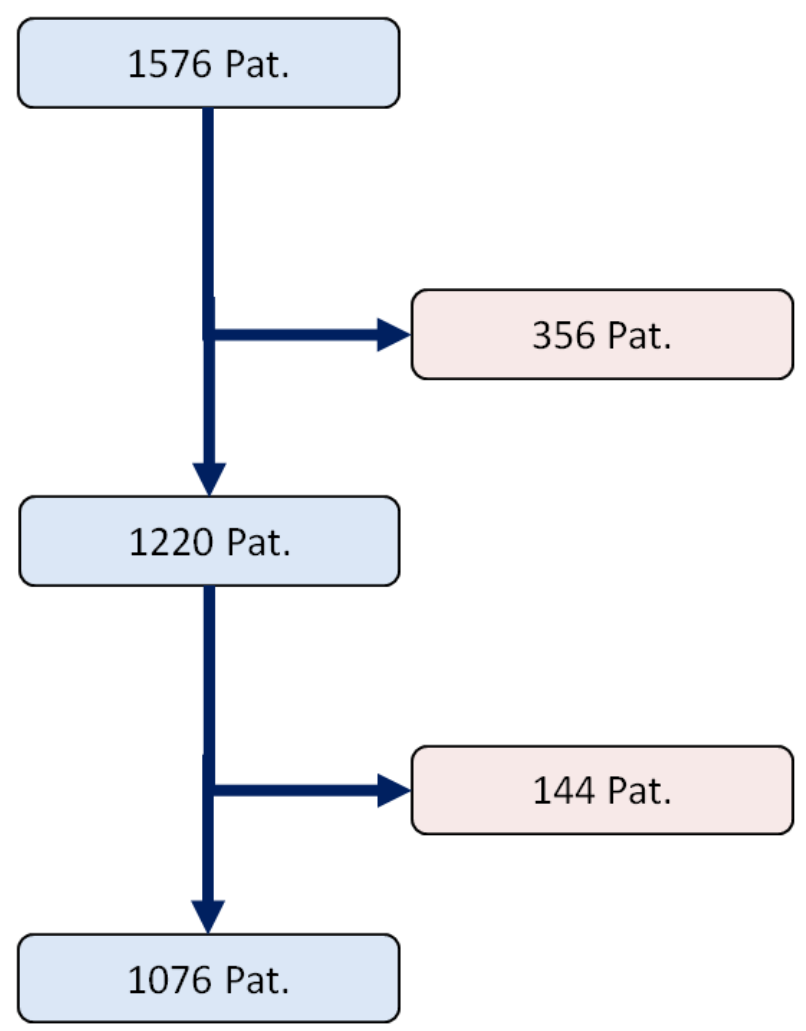

Ausschluss aller Patientinnen ohne operative Erstdiagnose oder mit unklaren Erstdiagnosedaten.

Ausschluss aller Patientinnen deren Endometrioseläsionen sich nicht in eine der 15 Gruppen bezüglich Art und Lokalisation einteilen ließen.

Patientenkollektiv 


\subsection{Populationen und Erstdiagnosefindung}

Bevor eine Auswertung vorgenommen werden konnte, musste eine einheitliche Definition der Erstdiagnose einer Endometriose gefunden werden. Zum besseren Verständnis wird in diesem Abschnitt kurz auf die Struktur der Datenbank und die Eingabeoberfläche des web-basierten Dokumentationssystems eingegangen.

Die Eingabe der Patientendaten geschieht visitenbasiert. Bei der Basisvisite werden alle bisherigen Daten bis zur Ersterfassung eingegeben. Nach der Basisvisite werden folgende Daten immer nur vom Zeitpunkt der letzten Visite bis zur nächsten Visite erfasst. Zur Dokumentation einer Diagnose einer Endometriose stehen pro Visite drei verschiedene Formulare zur Verfügung (siehe Fehler! Verweisquelle konnte nicht gefunden werden.).

\section{Abbildung 2: Eingabe-Formulare im web-basierten Dokumentationssystem}

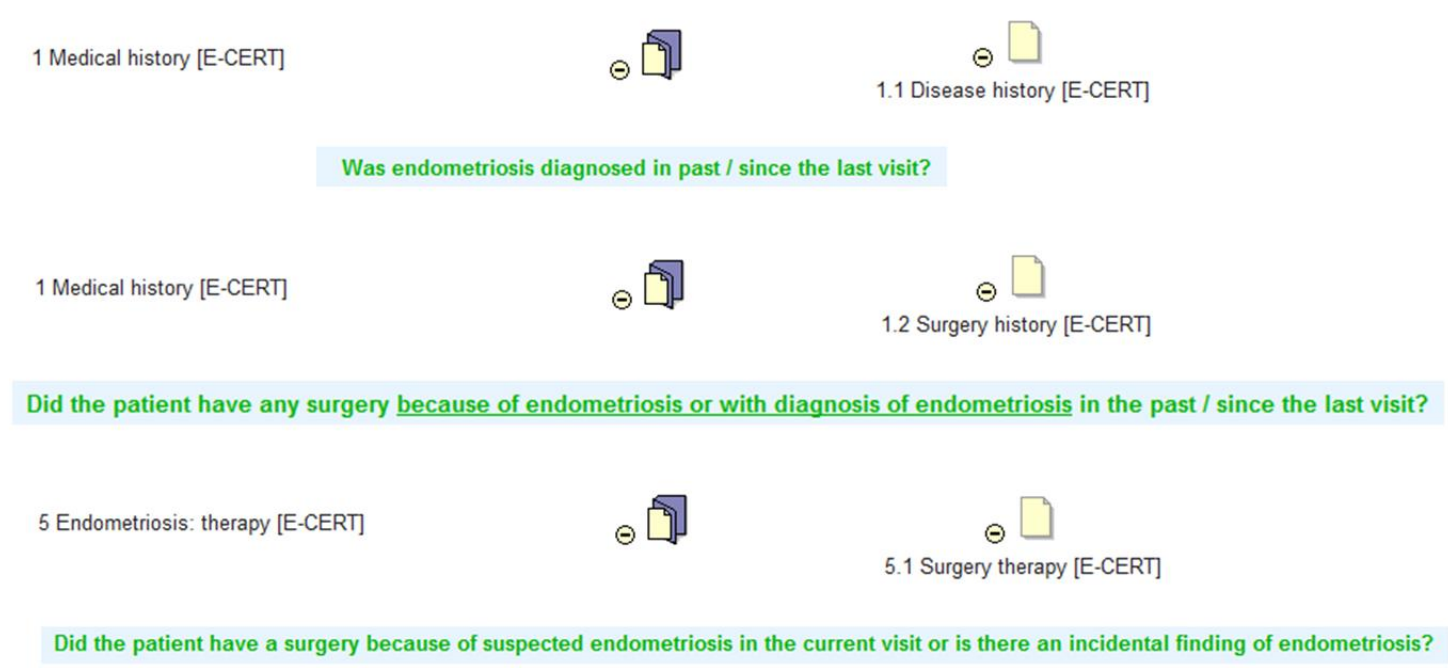

Disease history (anamnestische Diagnose): hier werden anamnestische Diagnosedaten erfasst. Pro Visite kann einmalig eine Angabe zu einer anamnestischen Erstdiagnose einer Endometriose getätigt werden. Es kann zudem genauer angegeben werden, wie die anamnestische Diagnose zustande gekommen ist. Es stehen die Optionen "Histologisch (in einer Operation)", "Makroskopisch (in einer Operation)", "Anamnestisch" und "Unbekannt" zur 
Verfügung. Die "Disease history" wird dann ausgefüllt, wenn in der Anamnese der Patientin die Diagnose einer Endometriose angegeben wird.

Surgery history (vergangene Operationen): in diesem Formular werden alle vergangenen Operationen dokumentiert, die wegen Verdacht auf eine Endometriose oder mit dem Ergebnis der Diagnose einer Endometriose durchgeführt wurden und von denen ein Operationsbericht vorliegt.

Surgery therapy (aktuelle Operationen): hier wird die aktuelle Operation dokumentiert, die wegen Verdacht auf eine Endometriose oder mit dem Ergebnis der Diagnose einer Endometriose durchgeführt wurde und von der ein Operationsbericht vorliegt. 
Aus diesen drei oben genannten Eingabemöglichkeiten wurde anschließend ein einheitliches Erstdiagnosedatum extrahiert.

\section{Abbildung 3: Erstellung der Patientenpopulation}

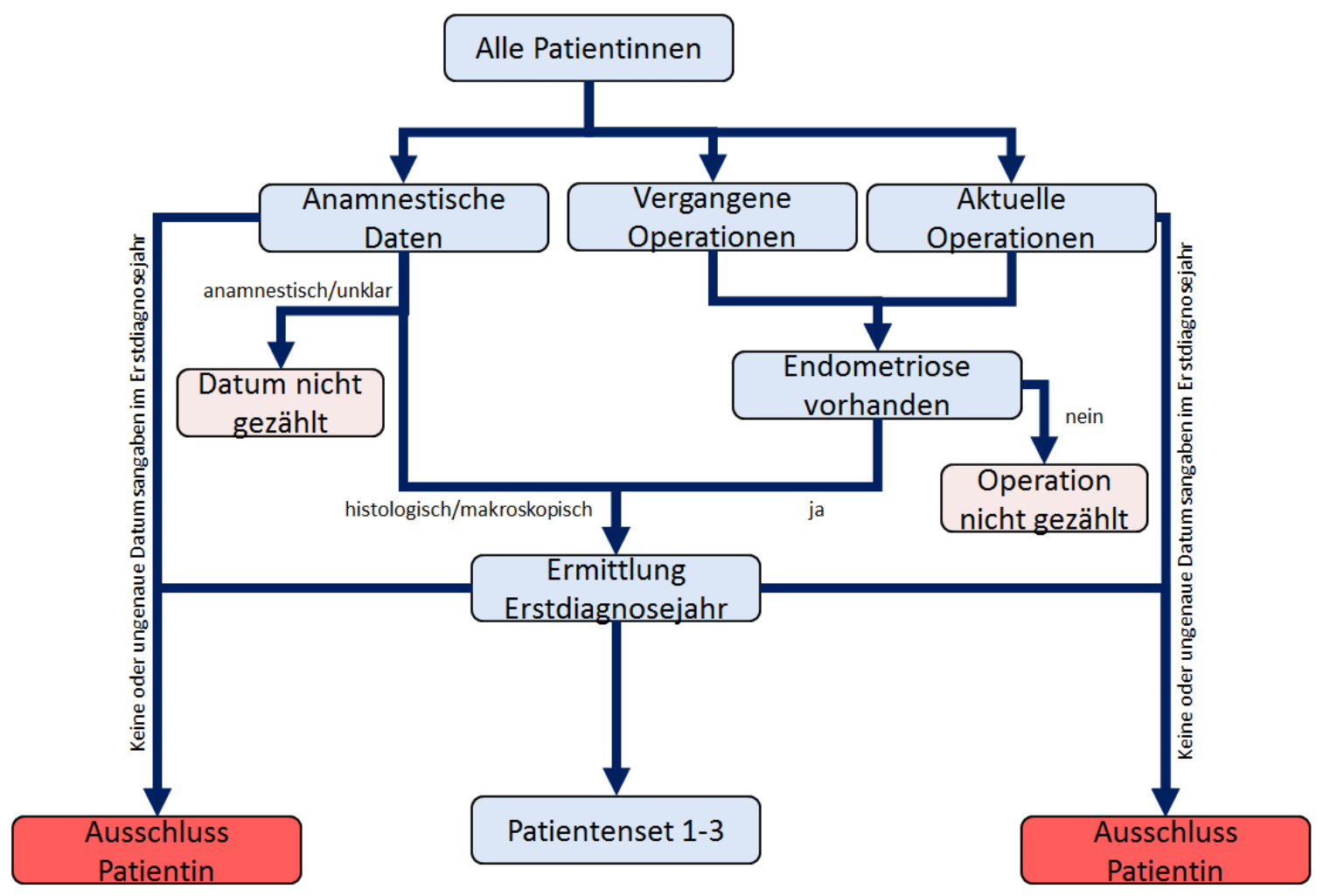

In einem ersten Schritt wurden alle anamnestischen Daten pro Patientin chronologisch geordnet. Nicht gezählt wurden alle anamnestischen Diagnosen, die nicht operativ oder histologisch waren.

Es folgte die chronologische Ordnung aller operativen Daten, bei denen eine Endometriose intraoperativ makroskopisch oder histologisch gesichert wurde. Je nach Population (Siehe Abschnitt 2.2.1- 2.2.3) wurde nun nach dem kleinsten in der Population erlaubten Diagnosedatum gesucht. Patientinnen, die im Erstdiagnosejahr eine ungenaue Datumsangabe hatten oder bei denen eine Diagnose einer Endometriose ohne Datumsangabe dokumentiert worden war, wurden von der weiteren Auswertung ausgeschlossen. Als ungenaue Datumsangabe wurde gewertet, wenn lediglich die Jahreszahl z.B. 2015 angegeben wurde. 
Es wurden drei Populationen für verschiedene Auswertungszwecke erstellt. Für die statistische Auswertung dieser Arbeit wurde lediglich die dritte Population verwendet. Die Erstellung der ersten beiden Populationen diente dem Verständnis der Daten.

\subsubsection{Population 1}

Die erste Population ist die umfangreichste, jedoch auch die mit der geringsten Datenqualität. Sie umfasste zum Zeitpunkt der Datenauswertung 1347 Patientinnen.

Diese Population kann wie folgt beschrieben werden: „Alle Patientinnen, die entweder eine dokumentierte Operation haben, bei der eine Endometriose festgestellt wurde oder bei denen in der Krankengeschichte dokumentiert wurde, dass eine Endometriose histologisch oder makroskopisch festgestellt wurde. Diagnosedatum ist hierbei das ältere der beiden Daten.“

Ausgeschlossen wurden alle Patientinnen, bei denen lediglich eine anamnestische Diagnose einer Endometriose vorlag, also keine makroskopische oder mikroskopische Diagnose. Zudem wurden alle Patientinnen ausgeschlossen, bei denen eine ungenaue Angabe im Erstdiagnosejahr vorlag. Als ungenaue Angabe wurde die fehlende Angabe von Monatsangaben gewertet, z.B. 2013.

\subsubsection{Population 2}

Die zweite Population bezieht sich ausschließlich auf operative Erstdiagnosen. Hier wurden anamnestische Erstdiagnosen ignoriert. Erfasst wurden nur Patientinnen, bei denen ein Operationsbericht der Erstdiagnose vorlag: "Alle Patientinnen, bei denen eine Operation dokumentiert wurde, in der eine Endometriose diagnostiziert wurde. Diagnosedatum ist hierbei das älteste Datum, welches bei einer Operation angegeben wurde. Falls in der Anamnese ein früheres Datum dokumentiert wurde, bleibt dies unberücksichtigt.“

$\mathrm{Da}$ in dieser Population rein anamnestische Erstdiagnosedaten ignoriert wurden, und es sich bei anamnestischen Daten oft um ungenaue 
Datumsangaben handelte, stieg die Anzahl der Patientinnen im Vergleich zur ersten Population auf 1432 Patientinnen.

\subsubsection{Population 3}

In der dritten Population gelten die strengsten Kriterien. Hier werden ebenfalls nur operative Erstdiagnosen erfasst, jedoch werden hier weitere mögliche Ungenauigkeiten durch eine anamnestische Diagnose verhindert. Einfach ausgedrückt handelt es sich hier um alle Patientinnen aus der Population 2, ohne all die Patientinnen aus Population 2, bei denen es ein Datum aus der Anamnese gibt, das vor dem Operationsdatum liegt.

Es ergibt sich somit die Definition "Alle Patientinnen, bei denen eine Operation dokumentiert wurde, in der eine Endometriose diagnostiziert wurde. Diagnosedatum ist hierbei das älteste Datum, welches bei einer Operation angegeben wurde. Ausgeschlossen werden alle Patientinnen, bei denen ein anamnestisches Datum für eine Endometriose vor dem möglichen operativen Erstdiagnosedatum liegt."

\subsubsection{Einteilung der Endometriosepatientinnen in Gruppen}

Bislang wurden entsprechend der Kriterien der Population 3 alle Patientinnen für die weitere Analyse ausgeschlossen (siehe Abbildung 1: Patientenkollektiv), bei denen in der Dokumentation der Operationen nicht eindeutig eine makroskopische oder mikroskopische Diagnose vorlag, das heißt alle Patientinnen, die eine anamnestische Diagnose vor der Erstdiagnose einer operativ gesicherten Endometriose hatten, sowie alle, bei denen im Erstdiagnosejahr mindestens einmal eine ungenaue Datumsangabe vorlag.

Zur weiteren Analyse musste nun die Endometriose nach Art und Lokalisation weiter unterteilt werden. Hierfür wurden 4 Gruppen (peritoneale Endometriose, ovarielle Endometriose/ Endometriom, solide tiefinfiltrierende Endometriose und Adenomyose), sowie 16 Untergruppen erstellt (siehe Tabelle 1: Gruppeneinteilung der Endometriosepatientinnen). 
Tabelle 1: Gruppeneinteilung der Endometriosepatientinnen

\begin{tabular}{|l|l|l|l|l|}
\hline Untergruppe & \multicolumn{1}{|c|}{ Peritoneal } & \multicolumn{1}{|c|}{ Ovariell } & $\begin{array}{c}\text { Solid } \\
\text { tiefinfiltrierend }\end{array}$ & Adenomyose \\
\hline 1 & Ja & Ja & Ja & Ja \\
\hline 2 & Ja & Ja & Ja & Nein \\
\hline 3 & Ja & Ja & Nein & Ja \\
\hline 4 & Ja & Ja & Nein & Nein \\
\hline 5 & Ja & Nein & Ja & Ja \\
\hline 6 & Ja & Nein & Ja & Nein \\
\hline 7 & Ja & Nein & Nein & Ja \\
\hline 8 & Ja & Nein & Nein & Nein \\
\hline 9 & Nein & Ja & Ja & Ja \\
\hline 10 & Nein & Ja & Ja & Nein \\
\hline 11 & Nein & Ja & Nein & Ja \\
\hline 12 & Nein & Ja & Nein & Nein \\
\hline 13 & Nein & Nein & Ja & Ja \\
\hline 14 & Nein & Nein & Ja & Nein \\
\hline 15 & Nein & Nein & Nein & Ja \\
\hline 16 & Nein & Nein & Nein & Nein \\
\hline
\end{tabular}

Bei weiteren 144 Patientinnen konnte keine eindeutige Einteilung der Endometriose nach Art und Lokalisation gemäß den Gruppen vorgenommen werden, so dass diese Patientinnen ebenfalls ausgeschlossen werden mussten. Die Untergruppe 16, bei der die Bedingungen für alle vier Gruppen (peritoneale, ovarielle, solid tiefinfiltrierende Endometriose und Adenomyose) nicht erfüllt sind, kam nicht vor. Die Zahl der Untergruppen reduzierte sich daher auf 15. Bei diesen 15 Untergruppen gab es eine eindeutige Zuordnung der Patientinnen zu jeweils nur einer Untergruppe.

Die Einteilung der 1076 Patientinnen in 15 Untergruppen war für eine Auswertung aufgrund der Gruppengröße nicht praktikabel. Die kleinste Gruppe enthielt zwei Patientinnen, drei weitere Gruppen jeweils vier, vier und sechs Patientinnen. In sieben Gruppen befanden sich weniger als 50 Patientinnen. Eine statistische Auswertung dieser Gruppen aufgrund einer fehlenden Power bei kleinen zu erwartenden Unterschieden bezüglich der zu untersuchenden Parameter erschien daher nicht sinnvoll, weshalb eine vereinfachte Einteilung angewendet wurde. 
Die neue Einteilung basierte auf den bereits oben genannten 15 Patientengruppen nach Art und Lokalisation der Endometriose, fasste allerdings kleinere Gruppen zusammen. Die Gruppe mit alleiniger peritonealer Endometriose mit 350 Patientinnen wurde als eigenständige Gruppe behandelt. Sie bildet somit "Gruppe 1: Peritoneal only" (Englisch für: ausschließlich peritoneal). Ebenfalls eine eigenständige Gruppe bildete die Gruppe mit peritonealer Endometriose und Adenomyose, sie wurde "Gruppe 2: Peritoneal and uterus" (Englisch für: Peritoneal und Uterus) genannt. Eine weitere eigenständige Gruppe war die Gruppe mit alleiniger Adenomyose, "Gruppe 3: Uterus only" (Englisch für: ausschließlich Gebärmutter). Vier weitere Gruppen wurden zu einer vierten Gruppe zusammengefasst. Diese umfasste alle Gruppen in der eine peritoneale Endometriose und eine tiefinfiltrierende Endometriose vorkam, unabhängig von einer Adenomyose oder einem Endometriom. Sie wurde "Gruppe 4: Peritoneal and TIE dominant" (Englisch für: Peritoneal und TIE dominant) genannt. Die letzte Gruppe umfasst alle anderen noch verbleibenden Gruppen. Sie beinhaltet Patientinnen mit peritonealer Endometriose und Endometriomen mit oder ohne Adenomyose, alleiniger Endometriome, alleiniger tiefinfiltrierender Endometriose, Endometriome mit tiefinfiltrierender Endometriose oder Adenomyose oder beiden und Patientinnen mit tiefinfiltrierender Endometriose und Adenomyose. Da diese Gruppe vorwiegend Patientinnen mit einem Endometriom beinhaltete, wurde die Gruppe "Gruppe 5: Endometrioma dominant" (Englisch für: Endometriom dominant) genannt. 


\subsection{Datenerhebung}

Im Rahmen einer jährlichen Zertifizierung durch die Stiftung EndometrioseForschung (SEF), die Europäische Endometriose Liga und die Endometriose Vereinigung Deutschland werden sämtliche Endometriosefälle in der Frauenklinik des Universitätsklinikums Erlangen durch eigens hierfür geschultes Personal erfasst. Die Dokumentation der Patientendaten in die IEEP Datenbank geschieht anhand der Patientenakten. In den Patientenakten sind klinische Informationen bezüglich Anamnese, Diagnostik, klinische Befunde, Therapie und histologische Ergebnisse enthalten (Burghaus et al., 2016). Die Patientendaten wurden in die Eingabeoberfläche des online-basierten Dokumentationssystems eingegeben. Die Eingabeoberfläche umfasste eine Vielzahl von Fragestellungen. Für die Auswertung im Rahmen dieser Arbeit sind jedoch nur wenige Fragen relevant. Sie werden im Folgenden aufgeführt.

\subsubsection{Diagnosefindung}

Wie bereits oben beschrieben wurde die Erstdiagnose aus verschiedenen Teilfragen generiert.

Unter Punkt 1.1.2 "Endometriosis disease history" im Formular 1.1 "Disease history" konnte das Datum der Erstdiagnose einer anamnestisch bekannten Endometriose eingegeben werden: "Was endometriosis diagnosed in past / since the last visit?" (siehe Abbildung 2: Eingabe-Formulare im web-basierten Dokumentationssystem).

Unter Punkt 1.2.2 "Endometriosis surgery history" im Formular 1.2 "Surgery history" konnten die Daten vergangener Operationen wegen Verdacht auf Endometriose oder mit der makroskopisch oder mikroskopisch gesicherten Endometriose eingegeben werden: "Did the patient have any surgery because of endometriosis or with diagnosis of endometriosis in the past / since the last visit?" (siehe Abbildung 2: Eingabe-Formulare im web-basierten Dokumentationssystem). 
Unter Punkt 5.1 "Surgery therapy" im gleichnamigen Formular konnten die Daten von aktuellen Operationen wegen Verdacht auf Endometriose oder mit der makroskopisch oder mikroskopisch gesicherten Endometriose eingegeben werden: "Did the patient have a surgery because of suspected endometriosis in the current visit or is there an incidental finding of endometriosis?" (siehe Abbildung 2: Eingabe-Formulare im web-basierten Dokumentationssystem).

Durch die Bejahung der Frage unter Punkt 1.2.2 und 5.1 öffnet sich ein Operationsformular "Surgical therapy", in dem sämtliche vergangene und aktuelle Operationen dokumentiert wurden. Hier konnte unter Punkt S.1.1 das Datum der Operation angegeben werden. Unter den Punkten S.2.1 und S.2.2 sollte zudem angegeben werden, ob eine peritoneale Endometriose oder eine tiefinfiltrierende Endometriose intraoperativ diagnostiziert wurde: "Was endometriosis diagnosed?" und "Was a deep infiltrating endometriosis diagnosed?" (Siehe Anhang: Anlage 1-5).

\subsubsection{Lokalisation und Art der Endometriose}

Wie bereits oben beschrieben, wurde die Lokalisation und Art der Endometriose aus verschiedenen Teilfragen generiert. Die Eingabe aktueller und vergangener Operationen geschah in einem eigens dafür vorgesehenen Formular "Surgical therapy", das über die Punkte 1.2.2 "Endometriosis surgery history" und 5.1 "Surgery therapy" in der Dokumentationsoberfläche aufgerufen werden konnte (Siehe Anhang: Anlage 1-5).

Unter Punkt S.2.1 konnte angegeben werden, ob eine peritoneale Endometriose diagnostiziert worden war: "Was endometriosis diagnosed?". Traf dies zu, öffnete sich ein Abschnitt, in dem die genaue Lokalisation der peritonealen Endometriose angegeben werden konnte. "Please describe the localisation of the endometriosis lesions.".

Unter dem Abschnitt "Peritoneum" fanden sich die Lokalisationen: "Fossa ovarica right"; "Fossa ovarica left"; "Douglas"; "Lig. Sacrouterinum right"; "Lig. Sacrouterinum left"; "Bladder"; "lleum", "Sigmoid", "Rectum"; "Middle abdomen"; "Upper abdomen". 
Als peritoneale Endometriose wurde jede Lokalisation im Abschnitt „Peritoneum" gewertet.

Im Freitextfeld mussten die Begriffe vorangehend sortiert werden. Zudem mussten Schreibfehler bei der Eingabe ausgeglichen werden.

Als peritoneale Endometriose wurden folgende Begriffe im Freitextfeld gewertet: *beck*; "paramet*; "pelvi*; *fundus uteri"; "latum*; uterus; *uterusserosa*; *uterusvorder*; *uterushinter*; " ${ }^{*}$ rotundum*; ${ }^{*}$ lig teres*; ${ }^{*}$ lig. teres"; ${ }^{*}$ teret"; *uterus-vw*; "uterus-hw*; "uterus $\mathrm{vw}^{*}$; "uterus $\mathrm{hw}^{*}$; " *sacral*; *uterin*; *leistenkan*; *iliaca externa*; *Hinterwand Uterus*; *Vorderwand Uterus*; ${ }^{*} \mathrm{HW}$ Uterus*; $\mathrm{VW}$ Uterus*; *uteri externa*; "uterus externa*; " ${ }^{*}$ corpus uteri*; ${ }^{*}$ Zervix ${ }^{*}$; ${ }^{*}$ rectouter*; ${ }^{*}$ rektouter"; ${ }^{*}$ rektovag*; ${ }^{*}$ rectovag*; "dougl*; " ${ }^{*}$ retrovag*; "sacrout*; "sakrout*; "zwerch*; "diaphra*; *perito*; "bauchdeck*; *bauchwand"; *nabel"; *omentum*; *Unterbauch*; *Abdomen*; " genitalis externa*; * genit.externa*; "darm*; "prärekt*; *praerekt";

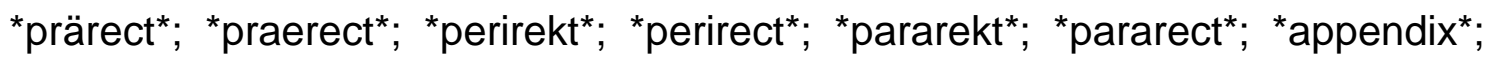

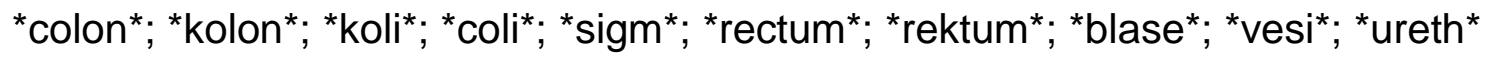
*ureter*; "Niere*; "nephr*.

Unter dem Abschnitt "Ovary" fanden sich die Lokalisationen: "Ovary right / Endometrioma"; "Ovary left / Endometrioma".

Unter dem Abschnitt "Other" befand sich: "Other". Hier konnte in einem Freitextfeld eine frei zu wählende Lokalisation eingegeben werden.

Als Endometriom wurde jede Lokalisation im Abschnitt „Ovary“ gewertet.

Als Endometriom wurden folgende Begriffe im Freitextfeld gewertet:

*tube*; *tuba*; *zyste*; *endometriom*; *fimbrien*; "salpin*; "ovary*; *ovar*; *adnex*.

Unter Punkt S.2.2 konnte eingegeben werden, ob eine tiefinfiltrierende Endometriose diagnostiziert wurde: "Was a deep infiltrating endometriosis diagnosed?". Traf dies zu, öffnete sich ein Abschnitt, in dem die Lokalisation der tiefinfiltrierenden Endometriose mithilfe des ENZIAN Scores angegeben werden konnte: "Please describe the localisation and the size in case of Enzian Score A-C in [mm] of deep infiltration endometriosis.". 
Es gab folgende Eingabemöglichkeiten: " $\mathrm{A}$ = Rectovaginal septum / vagina"; "Enzian A [mm]"; "B = Uterosacral ligaments, parametrium, pelvis"; "Enzian $B$ [mm]"; "C = Rectum / sigmoid"; "Enzian C [mm]"; "FA = Adenomyosis / uterus"; "FB = Bladder"; "FU = Ureter"; "FI = Bowel"; "FO = Other". Wurde "Other" angegeben, konnte die Lokalisation in einem Freitextfeld angegeben werden.

Als solide TIE wurde gewertet, wenn eine Angabe in den folgenden Feldern gemacht worden war: "A = Rectovaginal septum / vagina"; "Enzian A [mm]"; "B = Uterosacral ligaments, parametrium, pelvis"; "Enzian B [mm]"; "C = Rectum / sigmoid"; "Enzian C [mm]"; "FB = Bladder"; "FU = Ureter"; "FI = Bowel".

Als solide TIE wurden folgende Begriffe im Freitextfeld gewertet: "Zwerchfell*; *Zerchfell;; *Diaphrag*; *Diaprag*; "sectio*; *narbe*; *haut"; *Bauchnabel*; *subcutaneous*; *ureter*; *Niere*; *nephr*; "Peritoneum*; 'Endometriose extragenitalis'; "Bauchdecke*; " netz*; "omentum*; "Pelvis"; " pelvic*; "Becken*; "Douglas"; "tube*; "tuba*; " ${ }^{*} y s t{ }^{*}$; " ${ }^{*}$ endometriom*; ${ }^{*}$ fimbrien"; " ${ }^{*} a l p i{ }^{*}$; " ovary*; *ovar*; "adnex*; ; ocar"; "endometriosecyste*; "Blase*.

Als Adenomyose wurde gewertet, wenn eine Angabe im Feld „FA = Adenomyosis / uterus" gemacht wurde.

Als Adenomyose wurden folgende Begriffe im Freitextfeld der peritonealen Endometriose gewertet: "adenomyo*; *Adenoymo*; *Adenoyo*; *Adenyom*; *Adeomyos"; "Myometr"; *uteri interna*; "Uterusendometriose*; *UterusEndometriose*; *Uterus Endometriose*; *corpus em*; ${ }^{*}$ "corpus-em*; "corpusendomet"; "corpus-endometr"; "corpus endomet"; ${ }^{*}$ Adynomyosis*; *Adneomyosis*.

Als Adenomyose wurden folgende Begriffe im Freitextfeld der tiefinfiltrierenden Endometriose gewertet: "adenomyo*; *Adenoymo*; *Adenoyo*; *Adenyom*; *Adeomyos"; "Myometr"; *uteri interna*; *Uterusendometriose*; *UterusEndometriose*; *Uterus Endometriose*; ${ }^{*}$ corpus em*; ${ }^{*}$ corpus-em*; "corpusendomet"; " ${ }^{*}$ corpus-endometr"; ${ }^{*}$ corpus endomet"; ${ }^{*}$ Adynomyosis"; *Adneomyosis*.

Unter Punkt S.4.1 im Operationsformular „Endometriosis Surgery 1“ im Abschnitt S.4 "Histology“ konnte angegeben werden, ob eine Histologie durchgeführt worden war. Weiter konnte hier vermerkt werden, ob eine 
Adenomyose histologisch bestätigt worden war. "Adenomyosis confirmed“. Wurde eine Adenomyose histologisch bestätigt, wurde diese ebenfalls als Adenomyose gewertet.

\subsubsection{Alter bei Erstdiagnose}

Unter Punkt 0.1.1 im Formular 0.1. "Registration" konnte das Geburtsdatum eingegeben werden: "Date of birth" (Siehe Anhang: Anlage 6 und 7). Dies wurde zusammen mit dem errechneten Erstdiagnosedatum der Endometriose zum Alter bei Erstdiagnose gerechnet.

\subsubsection{Hauptvorstellungsgrund}

Unter Punkt 0.2.2 "What is the MAIN reason for consultation?" konnte der Hauptgrund der Vorstellung in dieser Visite angegeben werden: "Registration as type of case" (Siehe Anhang: Anlage 6 und 8).

Es bestanden hierbei folgende Eingabemöglichkeiten: "Women with abdominal / pelvic pain"; "Women with sub- and infertility"; "Women with other reason(s) for referral / admittance / surgery".

Die Angaben unter dem zuletzt genannten Punkt konnten genauer spezifiziert werden, diese Spezifikationen wurden allerdings in dieser Auswertung nicht berücksichtigt: "Other symptoms (as a hint of endometriosis)"; "Other diagnostic findings (suspicious for endometriosis)"; "Reason for surgery other than endometriosis (e.g. myoma, adhesions etc.)"; "Follow up (no symptoms)"; "Patient referred, but does not know why".

\subsubsection{Anzahl Schwangerschaften und Anzahl Lebendgeburten bei Erstvorstellung}

Unter Punkt 2.4.1 im Formular 2.4. "Pregnancies" konnte angegeben werden, ob die Patientin jemals schwanger gewesen war. "Has the patient been pregnant in past / since the last visit?" (Siehe Anhang: Anlage 9 und 10). 
Durch die Bejahung der Frage unter Punkt 2.4.1 öffnete sich ein Formular zur Eingabe der Schwangerschaften/Geburten "Pregnancy 1" (Siehe Anhang: Anlage 11).

Hier konnten verschiedene Angaben zu den einzelnen Schwangerschaften gemacht werden: "When ended the pregnancy?"; "In which pregnancy week ended the pregnancy?"; "NA"; "the patient is still pregnant"; "Birth weight in g"; "Weight unknown"; "NA"; "The pregnancy ended with".

Wurde eines dieser Felder im Formular für eine Schwangerschaft bis zur Basisvisite ausgefüllt, wurde dies als Schwangerschaft gezählt.

Die Anzahl der Lebendgeburten berechnete sich über das Eingabefeld: „The pregnancy ended with“.

Es fanden sich folgende Eingabemöglichkeiten: "Life birth"; "Death birth"; "Miscarriage"; "Abortion"; "Extrauteringravidity"; "Still pregnant"; "Life birth of gemini"; "Life birth of three or more children"; "Other".

"Life birth", "Life birth of gemini" und "Life birth of three or more children" wurden als Lebendgeburten gezählt.

\subsubsection{Gewicht und Größe}

Unter Punkt 2.1.2 "BMl” im Formular 2.1 "Lifestyle" konnte das Gewicht „What is the current weight in kg?" und die Größe „What is the current height in cm?" der Patientin angegeben werden. Aus den beiden Werten wurde der Body Mass Index (BMI) berechnet (Siehe Anhang: Anlage 9 und 12).

\subsubsection{Zykluslänge}

Unter Punkt 2.2.1 „Menstruation“ im Formular 2.2 „Reproductive Characteristics“ wurde die Zykluslänge eingegeben. „How many days does / did a menstrual cycle take from the first day of one bleeding to the first day of the next?" (Siehe Anhang: Anlage 9 und 13). 


\subsubsection{Alter bei Menarche}

Unter Punkt 2.2.1 "Menstruation" im Formular 2.2 "Reproductive Characteristics" war das Alter bei Menarche anzugeben. „Age at menarche“ (Siehe Anhang: Anlage 9 und 13).

\subsubsection{Schulbildung}

Unter Punkt 0.3.2 „Socio-economic status" im Formular 0.3 "Basic patient characteristics" konnte der Grad der schulischen Bildung angeben werden. "Specify the highest level of education“. Es war möglich, aus folgenden Kategorien auszuwählen: "No level of education or primary school"; "Hauptschule"; "Realschule"; "Gymnasium"; "High school"; "Professional"; "College / University"; "Graduation"; "Habilitation"; "Unknown" (Siehe Anhang: Anlage 6 und 14).

\subsection{Dokumentation und Datensicherheit}

Die Patientendaten aus den Patientenakten wurden in einem multizentrischen Online-Datenbanksystem erfasst. Die Dokumentation der Patientendaten erfolgte in einer Oracle-basierten Datenbank mittels eines elektronischen CaseReport-Formulars (eCRF). Dieses konnte online über einen Standardbrowser aufgerufen werden. Hierfür war keine weitere Softwareinstallation notwendig. Der Zugang zur Eingabe und Abfrage von Patientendaten war durch einen zugewiesenen Benutzernamen und ein selbst gewähltes Passwort geschützt. Die Eingabe der Daten im eCRF war visitenbasiert (Burghaus et al., 2016). Die Eingabe wurde durch ein eigens zur Dokumentation sämtlicher Endometriosefälle geschultes Personal vorgenommen.

\subsection{Statistische Auswertung}

Die Daten wurden mit einem online basierten Dokumentationssystems $\left(\right.$ secuTrial $\left.^{\circledR}\right)$ mithilfe einer für die Eingabe von Endometriosepatientinnen 
entwickelten Eingabeoberfläche unter www.myieep.de erfasst. Am 04.07.2017 wurden alle bis dahin in der Frauenklinik des Universitätsklinikums Erlangen dokumentierten Fälle mittels des von secuTrial ${ }^{\circledR}$ zur Verfügung gestellten Exporttools als VCS-Datei exportiert. Das Exporttool kann unter https://studien.uk-erlangen.de/apps/WebObjects/ST21-productive-

ExportSearchTool.woa/wa/choose?customer=IEEP1 aufgerufen werden.

In einem weiteren Schritt wurden die als VCS-Dateien zwischengespeicherten Patientendaten in eine Microsoft Access Datenbank Version 2010 importiert. In der Microsoft Access Datenbank wurden die oben genannten Berechnungen vorgenommen.

Die statistische Auswertung der Daten erfolgte in SPSS durch Herrn Prof. Dr. med. Peter Fasching und Herrn Simon Blum.

Die untersuchten Charakteristika des Patientenkollektivs wurden anhand von Mittelwerten, Standardabweichungen, absoluten Häufigkeiten und Prozentwerten beschrieben.

Untersucht wurden folgende Variablen: "Alter bei Erstdiagnose", "BMI bei Erstdiagnose", "Zykluslänge", "Anzahl der Schwangerschaften bei Erstvorstellung", "Anzahl der Lebendgeburten bei Erstvorstellung", "Alter bei Menarche", "Schulbildung" und der "Hauptvorstellungsgrund".

Die Variablen "Alter bei Erstvorstellung", "Anderes Outcome bei Schwangerschaft", "Blutungsdauer", Konsum von "rotem Fleisch" und "weißem Fleisch", Familienanamnese bezüglich "Endometriose", "Endometriumkarzinom" und "Ovarialkarzinom", "Symptomatik wie "Pelvine Schmerzen", "Dyspareunie", "Dyschezie", "Dysurei" wurden für die weitere Auswertung nicht berücksichtigt, da diese Fragestellungen durch zu wenige Patientinnen beantwortet worden waren.

Für die Untersuchung des Zusammenhangs zwischen den fünf oben beschriebenen Gruppen (Siehe 2.2.4 Einteilung der Endometriosepatientinnen in Gruppen) und dem Alter bei Erstdiagnose wurden die Mittelwerte des Alters bei Erstdiagnose innerhalb der Gruppen mittels einfaktorieller ANOVA verglichen und der dazugehörige $p$-Wert berechnet. Als abhängige Variable wurde das Alter bei Erstdiagnose angenommen. Es konnte von einer 
Normalverteilung ausgegangen werden.

Analog wurde bei der Auswertung der Variablen "BMI bei Erstvorstellung", "Zykluslänge bei Erstvorstellung" und "Alter bei Menarche" vorgegangen. Hier wurde ebenfalls jeweils von einer Normalverteilung ausgegangen.

Zur Berechnung des Einflusses der Variablen "Vorstellungsgrund bei Erstvorstellung", "Anzahl Schwangerschaften bei Erstvorstellung", "Anzahl Lebendgeburten bei Erstvorstellung" und "Schulbildung bei Erstvorstellung" wurde der Chi-Quadrat-Test herangezogen.

Die Variablen wurden mittels Chi-Quadrat-Test jeweils auf eine Normalverteilung innerhalb der fünf Gruppen untersucht. Hierfür wurden die Variablen "Schwangerschaften", "Lebendgeburten" und "Schulbildung bei Erstvorstellung" in Kategorien zusammengefasst. Es wurde eine Kategorie für 0,1 oder $\geq 2$ Schwangerschaften beziehungsweise Lebendgeburten gebildet. Bei der Schulbildung wurden zwei Kategorien gebildet. Die Variablen "College/University“, „Graduation“, „Gymnasium“, „Habilitation“ und „High school" wurden zur Kategorie "Hochschulreife" und die Variablen „Hauptschule“, „Professional“ und „Realschule“ wurden zu "NichtHochschulreife" zusammengefasst.

Der berechnete $p$-Wert sagte aus, ob sich der Korrelationskoeffizient signifikant von 0 unterscheidet oder nur eine zufällige Abweichung darstellt. Alle Tests waren zweiseitige Tests und ein $p$-Wert $<0,05$ wurde als statistisch signifikant betrachtet. 


\section{Ergebnisse}

\subsection{Patientencharakteristika}

Es wurden bis zum 04.07.2017 insgesamt 1576 Patientinnen erfasst. Von diesen Patientinnen wurden 356 Patientinnen ausgeschlossen, bei denen kein geeignetes Erstdiagnosedatum gefunden werden konnte. Von den verbleibenden 1220 Patientinnen mit einer operativen Erstdiagnose wurden weitere 144 Patientinnen ausgeschlossen, bei denen sich keine eindeutige Art und Lokalisation der Endometriose-Läsionen anhand der 16 definierten Untergruppen bestimmen lies. Für die Auswertung in dieser Arbeit ergab sich ein Kollektiv von 1076 Patientinnen mit einer operativen Erstdiagnose einer Endometriose.

Für die Auswertung wurde die Basisvisite untersucht. Pro Patientin wurde demnach die erste Visite, mit der die Patientin dokumentiert wurde, mit den bis dahin bekannten Daten ausgewertet. Es wurden lediglich operative Fälle für die Auswertung herangezogen. Es handelte sich beim hier dargestellten Kollektiv um Patientinnen, bei denen erstmalig intraoperativ in der Frauenklinik des Universitätsklinikums Erlangen eine Endometriose makroskopisch oder histologisch gesichert worden war oder bei denen in der aktuellen Operation ein Rezidiv makroskopisch oder histologisch gesichert worden war.

Die untersuchten Patientencharakteristika sind in Tabelle 2: Patientencharakteristika der untersuchten Patientenpopulation abgebildet und sollen im Folgenden erläutert werden. 
Tabelle 2: Patientencharakteristika der untersuchten Patientenpopulation

\begin{tabular}{|c|c|c|}
\hline Patientencharakteristika & Mittelwert / Frequenzen & $\begin{array}{c}\text { Standardabweichung / } \\
\text { Prozent }\end{array}$ \\
\hline Gesamt & 1076 & 100 \\
\hline Alter bei Erstdiagnose & 33,86 & 8,423 \\
\hline BMI bei Erstvorstellung & 24,138 & 5,1362 \\
\hline Zykluslänge & 28,56 & 9,038 \\
\hline $\begin{array}{l}\text { Schwangerschaft bei } \\
\text { Erstvorstellung }\end{array}$ & 0,68 & 1,076 \\
\hline $\begin{array}{l}\text { Lebendgeburten bei } \\
\text { Erstvorstellung }\end{array}$ & 0,46 & 0,830 \\
\hline Alter bei Menarche & 12,99 & 1,507 \\
\hline \multicolumn{3}{|l|}{ Schulbildung } \\
\hline Hochschule/Universität & 74 & 6,9 \\
\hline Graduation & 28 & 2,6 \\
\hline Gymnasium & 28 & 2,6 \\
\hline Habilitation & 8 & 0,7 \\
\hline Hauptschule & 17 & 1,6 \\
\hline High school & 4 & 0,4 \\
\hline Lehrberuf & 190 & 17,7 \\
\hline Realschule & 36 & 3,3 \\
\hline keine Angabe & 691 & 64,2 \\
\hline \multicolumn{3}{|l|}{ Hauptvorstellungsgrund } \\
\hline Schmerzen & 520 & 48,3 \\
\hline Infertilität & 295 & 27,4 \\
\hline $\begin{array}{l}\text { Andere Gründe für eine } \\
\text { Operation als Endometriose }\end{array}$ & 214 & 19,9 \\
\hline Andere diagnostische Befunde & 38 & 3,5 \\
\hline Andere: Follow Up & 3 & 0,3 \\
\hline Andere Symptome & 2 & 0,2 \\
\hline Andere nicht näher bezeichnet & 1 & 0,1 \\
\hline keine Angaben & 3 & 0,3 \\
\hline
\end{tabular}


Das Durchschnittsalter der Patientinnen lag zum Zeitpunkt der Erstdiagnose bei 33,86 Jahren (Standardabweichung $\pm 8,423$ ). In dieser Auswertung gab es keine fehlenden Daten. Für die Auswertung des BMI lagen bei 813 Patientinnen auswertbare Daten vor. Hier ergab sich ein BMI an der oberen Norm mit 24,14 (Standardabweichung $\pm 5,1362$ ). Daten für die Auswertung der Zykluslänge lagen bei 600 Patientinnen vor. Es ergab sich ein Mittelwert von 28,56 Tagen (Standardabweichung $\pm 9,038$ ). Deutlich häufiger lagen die Angaben für die Berechnung der Anzahl der Schwangerschaften und Lebendgeburten vor. 1069 Patientinnen hatten durchschnittlich 0,68 Schwangerschaften (Standardabweichung 1,076) und 1067 Patientinnen hatten durchschnittlich 0,46 Lebendgeburten (Standardabweichung $\pm 0,830$ ) zum Zeitpunkt der Erstvorstellung. Bei 991 Patientinnen ergab sich ein durchschnittliches Alter bei Menarche von 12,99 Jahren (Standardabweichung $\pm 1,507$ ). Angaben zur Schulbildung lagen bei 691 Patientinnen vor, wohingegen nur bei 3 Patientinnen kein Hauptvorstellungsgrund für die Erstvisite angegeben worden war. Genauere Angaben bezüglich der Schulbildung und des Hauptvorstellungsgrundes finden sich in der Tabelle 2: Patientencharakteristika der untersuchten Patientenpopulation.

Gruppenübergreifend waren 891 Patientinnen $(82,8 \%)$ an einer peritonealen Endometriose erkrankt. 291 Patientinnen $(27,0 \%)$ hatten ein Endometriom. Eine Adenomyose lag bei 404 Patientinnen (37,5\%) und eine tiefinfiltrierenden Endometriose nur bei 299 Patientinnen (27,8 \%) vor. Da Patientinnen an mehreren Formen der Endometriose erkrankt sein konnten, und somit keine eindeutige Zuordnung der Patientin zu einer der vier genannten EndometrioseFormen erfolgen konnte, wurde, wie oben bereits in Abschnitt 2.2.4 erläutert, eine eindeutige Einteilung in fünf Gruppen gewählt. Mithilfe dieser Einteilung konnte jede Patientin eindeutig einer Gruppe zugeordnet werden. 
Tabelle 3: Einteilung der fünf Patientengruppen

\begin{tabular}{|l|r|r|}
\hline \multicolumn{1}{|c|}{ Endometriose-Lokalisation } & N & Prozent \\
\hline Gruppe 1: Peritoneal only & 350 & 32,5 \\
Peritoneale Endometriose / TIE nein & 142 & \\
\hline Gruppe 2: Peritoneal and uterus & & 13,2 \\
Peritoneale Endometriose / Adenomyose & 115 & \\
\hline Gruppe 3: Uterus only & & 10,7 \\
Endometriose nein / Adenomyose & 105 & \\
\hline Gruppe 4: Peritoneal and TIE dominant & 60 & 9,8 \\
Peritoneale Endometriose / solide TIE & 57 & 5,6 \\
Peritoneale Endometriose und Endometriom / solide TIE & 53 & 5,3 \\
Peritoneale Endometriose / solide TIE und Adenomyose & & 4,9 \\
Peritoneale Endometriose und Endometriom / solide TIE und & & \\
Adenomyose & & \\
\hline Gruppe 5: Endometrioma dominant & 99 & 9,2 \\
Peritoneale Endometriose und Endometriom / TIE nein & 40 & 3,7 \\
Endometriom / TIE nein & 25 & 2,3 \\
Peritoneale Endometriose und Endometriom / Adenomyose & 14 & 1,3 \\
Endometriose nein / solide TIE & 6 & 0,6 \\
Endometriom / Adenomyose & 4 & 0,4 \\
Endometriom / solide TIE & 4 & 0,4 \\
Endometriom / solide TIE und Adenomyose & 2 & 0,2 \\
Endometriose nein / solide TIE und Adenomyose & 1076 & 100,0 \\
\hline Gesamt & & \\
\hline
\end{tabular}

Die Gruppe 1 mit ausschließlich peritonealer Endometriose umfasste 350 Patientinnen, die Gruppe 2 mit peritonealer Endometriose und Adenomyose umfasste 142. Die kleinste Gruppe mit 115 Patientinnen, Gruppe 3, war die Gruppe mit den Patientinnen, die ausschließlich an einer Adenomyose erkrankt waren. Die Gruppe 4 umfasste mit 275 Patientinnen alle Patientinnen, die an einer peritonealen Endometriose und einer tiefinfiltrierenden Endometriose erkrankt waren, unabhängig davon, ob sie zusätzlich noch an einer Adenomyose oder einem Endometriom erkrankt waren. In der Gruppe 5, mit 194 Patientinnen, befanden sich alle Patientinnen mit einer peritonealen Endometriose und Endometriom mit oder ohne Adenomyose, alleiniger Endometriome, alleiniger tiefinfiltrierender Endometriose, Endometriome mit 
tiefinfiltrierender Endometriose und/oder Adenomyose und Patientinnen mit tiefinfiltrierender Endometriose und Adenomyose. Die Abbildung 4: Anzahl Patientinnen pro Gruppe zeigt die Verteilung der Patientinnen auf die fünf Gruppen.

\section{Abbildung 4: Anzahl Patientinnen pro Gruppe}

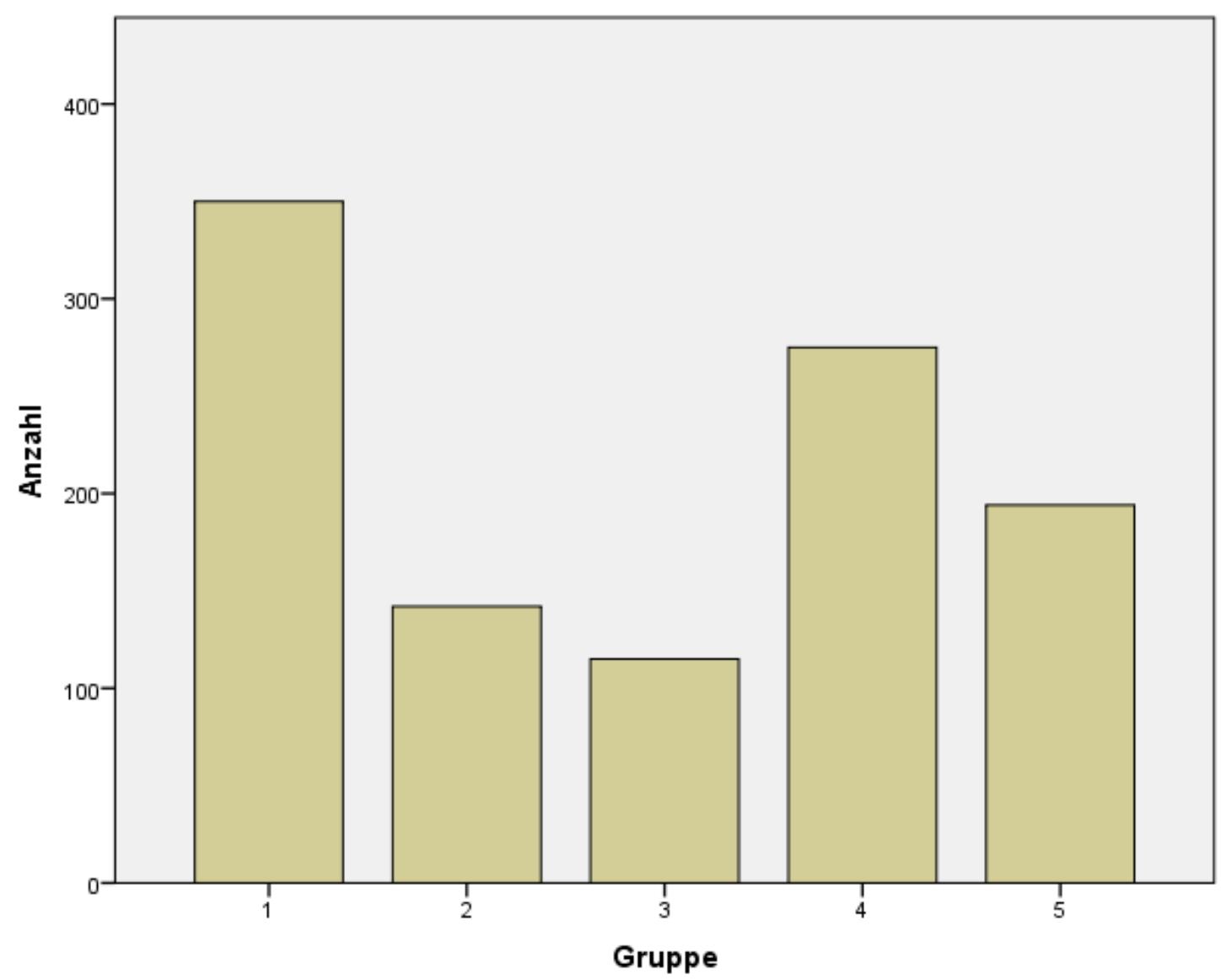

In der Tabelle 4: Patientencharakteristika in den fünf Gruppen sind die Frequenzen und dazugehörigen Prozentangaben für die Variablen "Schwangerschaften", "Lebendgeburten", "Schulbildung" und "Vorstellungsgrund", sowie die Mittelwerte und Standardabweichung für die Variablen "Alter bei Erstdiagnose", "BMI", "Zykluslänge" und "Alter bei Menarche" pro Gruppe dargestellt. 
Tabelle 4: Patientencharakteristika in den fünf Gruppen

\begin{tabular}{|c|c|c|c|c|c|c|c|c|c|c|}
\hline & \multicolumn{2}{|c|}{$\begin{array}{c}\text { Gruppe } 1 \\
\text { Peritoneal only }\end{array}$} & \multicolumn{2}{|c|}{$\begin{array}{c}\text { Gruppe } 2 \\
\text { Peritoneal and } \\
\text { uterus }\end{array}$} & \multicolumn{2}{|c|}{$\begin{array}{c}\text { Gruppe } 3 \\
\text { Uterus only }\end{array}$} & \multicolumn{2}{|c|}{$\begin{array}{c}\text { Gruppe } 4 \\
\text { Peritoneal and } \\
\text { TIE dominant }\end{array}$} & \multicolumn{2}{|c|}{$\begin{array}{c}\text { Gruppe } 5 \\
\text { Endometrioma } \\
\text { dominant }\end{array}$} \\
\hline & $\begin{array}{l}\text { Mittelwert / } \\
\text { Frequenz }\end{array}$ & $\begin{array}{c}\text { Standardabw. } \\
\text { / Prozent }\end{array}$ & $\begin{array}{l}\text { Mittelwert / } \\
\text { Frequenz }\end{array}$ & $\begin{array}{c}\text { Standardabw. } \\
\text { / Prozent }\end{array}$ & $\begin{array}{l}\text { Mittelwert / } \\
\text { Frequenz }\end{array}$ & $\begin{array}{c}\text { Standardabw. } \\
\text { / Prozent }\end{array}$ & $\begin{array}{l}\text { Mittelwert / } \\
\text { Frequenz }\end{array}$ & $\begin{array}{c}\text { Standardabw. } \\
\text { / Prozent }\end{array}$ & $\begin{array}{l}\text { Mittelwert / } \\
\text { Frequenz }\end{array}$ & $\begin{array}{l}\text { Standardabw. } \\
\text { / Prozent }\end{array}$ \\
\hline Fälle & 350 & 32,5 & 142 & 13,2 & 115 & 10,7 & 275 & 25,6 & 194 & 18,0 \\
\hline Alter bei ED & 32,64 & 9,139 & 33,28 & 8,131 & 35,90 & 9,887 & 34,28 & 7,243 & 34,99 & 7,471 \\
\hline BMI & 24,039 & 5,4615 & 24,507 & 5,0952 & 24,282 & 4,7487 & 23,993 & 5,1710 & 24,226 & 4,7300 \\
\hline Zykluslänge & 28,08 & 5,210 & 29,10 & 8,462 & 30,43 & 14,167 & 27,65 & 3,294 & 29,35 & 14,955 \\
\hline Alter bei Menarche & 12,98 & 1,447 & 13,01 & 1,667 & 12,77 & 1,635 & 13,09 & 1,438 & 12,97 & 1,503 \\
\hline \multicolumn{11}{|l|}{ Schwangerschaft } \\
\hline 0 Schwangerschaft & 229 & 21,4 & 82 & 7,7 & 48 & 4,5 & 186 & 17,4 & 125 & 11,7 \\
\hline 1 Schwangerschaft & 55 & 5,1 & 34 & 3,2 & 20 & 1,9 & 51 & 4,8 & 30 & 2,8 \\
\hline$\geq 2$ Schwangerschaften & 62 & 5,8 & 25 & 2,3 & 45 & 4,2 & 38 & 3,6 & 39 & 3,6 \\
\hline \multicolumn{11}{|l|}{ Lebendgeburten } \\
\hline 0 Lebendgeburt & 259 & 24,3 & 102 & 9,6 & 60 & 5,6 & 211 & 19,8 & 137 & 12,8 \\
\hline 1 Lebendgeburt & 44 & 4,1 & 18 & 1,7 & 18 & 1,7 & 36 & 3,4 & 28 & 2,6 \\
\hline$\geq 2$ Lebendgeburten & 42 & 3,9 & 21 & 2,0 & 34 & 3,2 & 28 & 2,6 & 29 & 2,7 \\
\hline \multicolumn{11}{|l|}{ Schulbildung } \\
\hline Hochschulreife & 52 & 13,5 & 15 & 3,9 & 8 & 2,1 & 42 & 10,9 & 25 & 6,5 \\
\hline $\begin{array}{l}\text { Keine } \\
\text { Hochschulreife }\end{array}$ & 80 & 20,8 & 29 & 7,5 & 22 & 5,7 & 62 & 16,1 & 50 & 13,0 \\
\hline \multicolumn{11}{|l|}{ Vorstellungsgrund } \\
\hline Schmerzen & 166 & 15,5 & 73 & 6,8 & 67 & 6,2 & 129 & 12,0 & 85 & 7,9 \\
\hline Infertilität & 87 & 8,1 & 45 & 4,2 & 24 & 2,2 & 85 & 7,9 & 54 & 5,0 \\
\hline Andere Gründe & 95 & 8,9 & 24 & 2,2 & 24 & 2,2 & 61 & 5,7 & 54 & 5,0 \\
\hline
\end{tabular}




\subsection{Einfaktorielle Varianzanalyse (ANOVA)}

Das Patientenkollektiv wurde nun dahingehend untersucht, ob es einen Zusammenhang zwischen dem Alter bei Erstdiagnose und der Art und Lokalisation einer Endometriose anhand der bereits beschriebenen fünf Patientengruppen besteht. Hierfür wurde eine einfaktorielle Varianzanalyse mittels ANOVA-Test durchgeführt. Die Mittelwerte der Variable "Alter bei Erstdiagnose" als abhängige Variable wurden hinsichtlich signifikanter Unterschiede zwischen den Gruppen untersucht. Die Gruppen galten entsprechend der Voraussetzung für den ANOVA-Test als unabhängige Variable. Die Testung erfolgte an allen 1076 Patientinnen.

Das durchschnittliche Alter bei Erstdiagnose unterschied sich zwischen den Gruppen nur um wenige Jahre. Am jüngsten waren die Patientinnen in der Gruppe 1 mit ausschließlich peritonealer Endometriose mit 32,46 Jahren. Demgegenüber standen die Patientinnen in der Gruppe 3 mit alleiniger Adenomyose mit 35,90 Jahren. Die Gruppe 2 war im Durchschnitt 33,28 Jahre, die Gruppe 4 im Durchschnitt 34,28 Jahre und die Gruppe 5 im Durchschnitt 34,99 Jahre alt (siehe Tabelle 4: Patientencharakteristika in den fünf Gruppen). In der einfaktoriellen Varianzanalyse ergab sich trotz der relativ geringen Altersunterschiede ein hoch signifikanter Unterschied zwischen den Gruppen mit einem $P$-Wert von $<0,001$. Aus den Daten lässt sich herauslesen, dass ein signifikanter Zusammenhang zwischen dem durchschnittlichen Alter bei Erstdiagnose und der Art und Lokalisation entsprechend der fünf Gruppen bestand. 


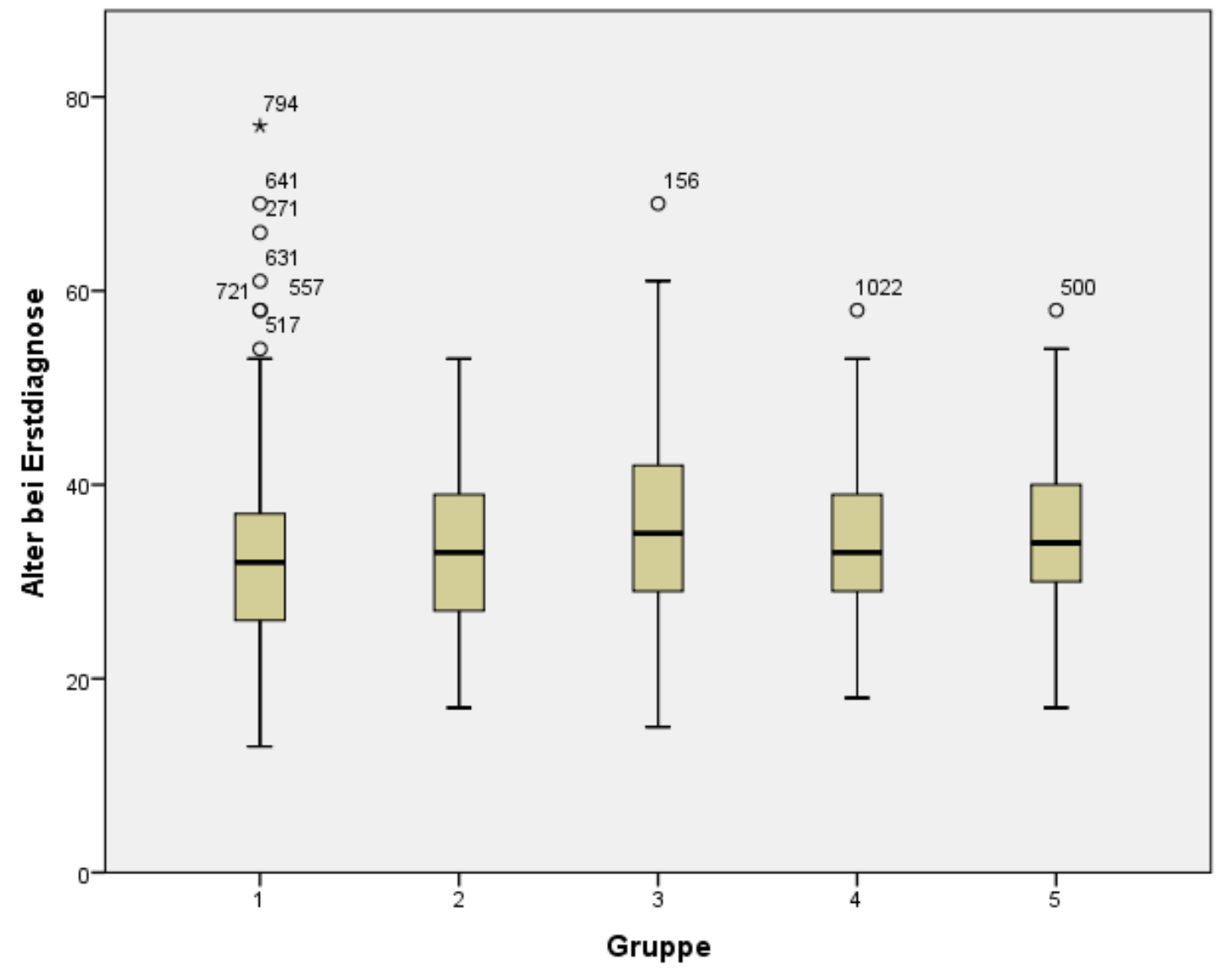

Analog hierzu wurden auch die Variablen "BMI", "Zykluslänge" und "Alter bei Menarche" mittels einfaktorieller Varianzanalyse (ANOVA) untersucht. Die untersuchten Parameter "BMI" (813 Patientinnen), "Zykluslänge" (600 Patientinnen) und "Alter bei Menarche" (991 Patientinnen) galten jeweils als abhängige Parameter, während die Gruppen als unabhängige Parameter angenommen wurden. Hierbei zeigte sich für alle drei Variablen kein signifikantes Ergebnis. Diese Daten wiesen darauf hin, dass es keinen Zusammenhang zwischen dem "BMI", "Zykluslänge" oder "Alter bei Menarche" und der Art und Lokalisation der Endometriose anhand der fünf definierten Gruppen gibt. 


\subsection{Chi-Quadrat-Test und Kreuztabellen}

Das Patientenkollektiv wurde nun dahingehend untersucht, ob es einen Zusammenhang zwischen dem Vorstellungsgrund und der Art und Lokalisation einer Endometriose anhand der fünf vorangehend definierten Patientengruppen besteht. Hierfür wurde eine Testung mittels Chi-Quadrat-Test durchgeführt. Der Vorstellungsgrund konnte drei Ausprägungen annehmen: "Schmerz", "Infertilität" und "anderer Vorstellungsgrund". Da sowohl die fünf Gruppen wie auch der zu untersuchende Parameter "Vorstellungsgrund" als kategoriale Variable vorlagen, wurde der Chi-Quadrat-Test zur Untersuchung auf Zusammenhänge herangezogen. Untersucht wurden 1073 Patientinnen, das waren 99,7\% des ursprünglichen Patientenkollektivs. Drei Patientinnen wurden aufgrund fehlender Angaben von der Testung ausgeschlossen. Es zeigte sich mit einem P-Wert von 0,62 kein signifikanter Zusammenhang zwischen dem Vorstellungsgrund und den fünf Gruppen.

Analog zu dieser Fragestellung wurden auch die Schwangerschaftsraten anhand dreier Kategorien: "0 Schwangerschaften", "1 Schwangerschaft" und " $\geq$ 2 Schwangerschaften" untersucht. Von den 1076 Patientinnen mussten sieben Patientinnen aufgrund fehlender Angaben von der Untersuchung ausgeschlossen werden. Die Testung erfolgte an 1069 Patientinnen (99,3\%). Es zeigte sich ein hoch signifikantes Ergebnis mit einem P-Wert von $<0,001$. Die Frequenzen sind im folgenden Diagramm (Abbildung 6: Verteilung der Schwangerschaften pro Gruppe wiedergegeben. 
Abbildung 6: Verteilung der Schwangerschaften pro Gruppe

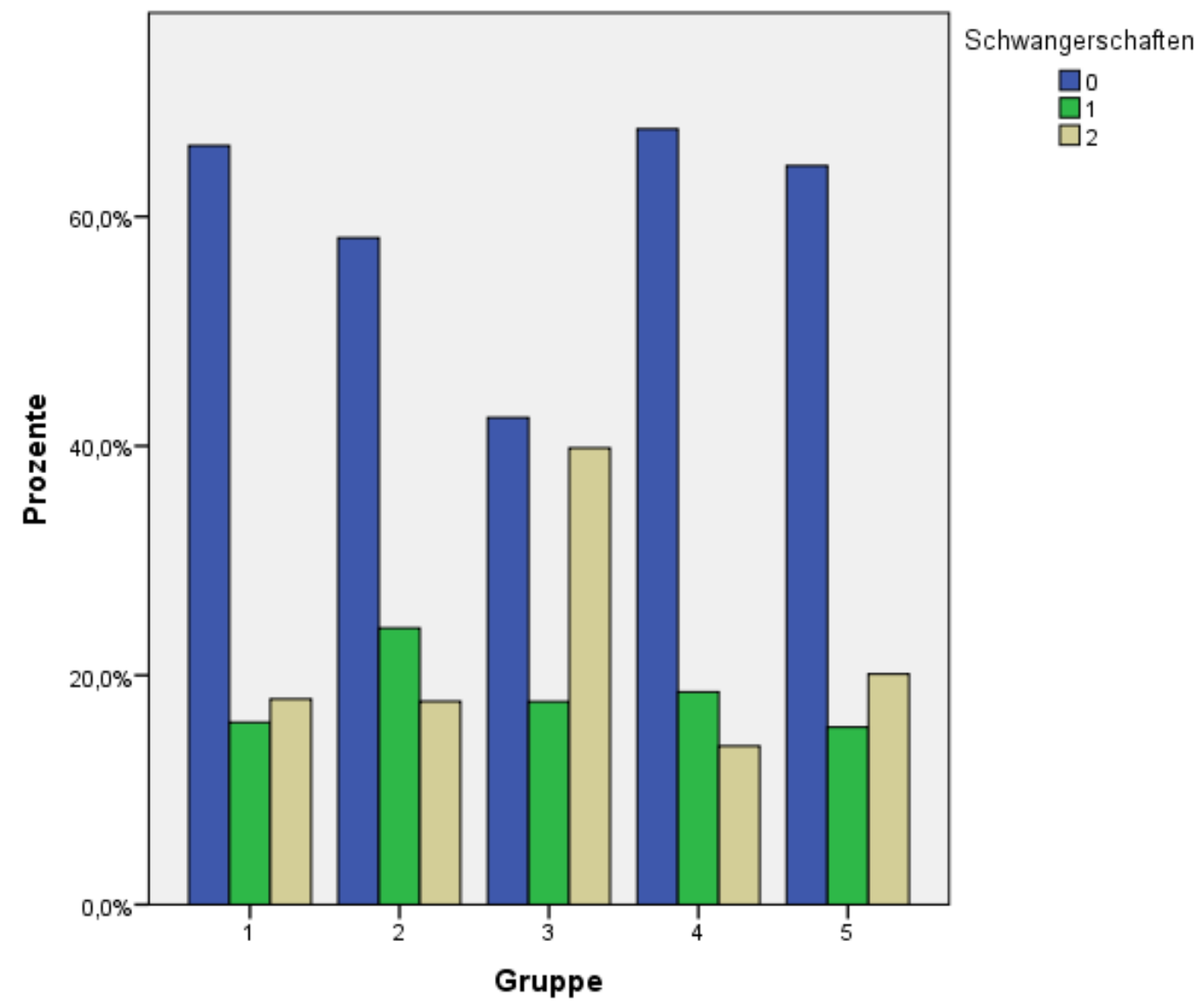

Ein ähnliches Ergebnis zeigte sich bei der Untersuchung der Lebendgeburtenraten mittels Chi-Quadrat-Test. Die Anzahl der Lebendgeburten wurden ebenfalls in drei Kategorien unterteilt: "0 Lebendgeburten", "1 Lebendgeburt" und " $\geq 2$ Lebendgeburten". Aufgrund fehlender Daten wurden hier neun Patientinnen von der Testung ausgeschlossen. Die Testung erfolgte an 1067 Patientinnen (99,2 \%) mit gültigen Angaben. Es zeigte sich wie schon bei den Schwangerschaftsraten ein hoch signifikantes Ergebnis mit einem PWert von <0,001. Die Frequenzen der Lebendgeburten sind im folgenden Diagramm (Abbildung 7: Verteilung der Lebendgeburten pro Gruppe) dargestellt. 


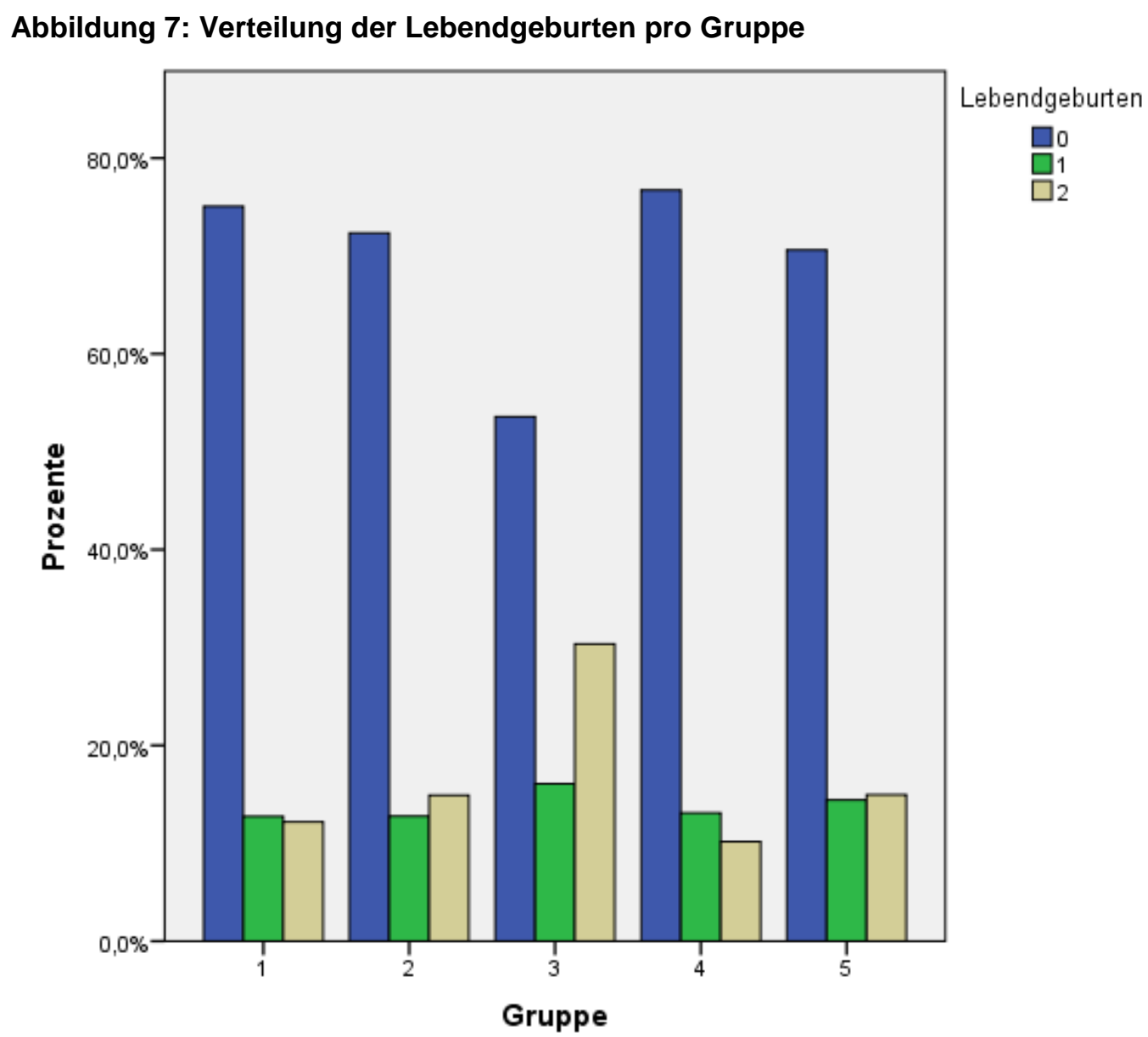

Als letzte Untersuchung wurde der Zusammenhang zwischen der Schulbildung und den fünf Gruppen mittels Chi-Quadrat-Test untersucht. Die Schulbildung wurde in zwei Kategorien aufgeteilt. Die erste Kategorie umfasste alle Patientinnen ohne Hochschulreife, die zweite Kategorie alle Patientinnen mit einer Hochschulreife. Aufgrund eines unzureichenden Ausfüllstatus mussten von den ursprünglich 1076 Patientinnen 691 Patientinnen von der Testung ausgeschlossen werden. Beim verbleibenden Patientenkollektiv von 385 Patientinnen ergab sich mit einem P-Wert von 0,591 kein signifikantes Ergebnis. Die Daten wiesen auf einen fehlenden Zusammenhang zwischen Schulbildung und Endometriose hin. 


\section{Diskussion}

Bei unseren Daten zeigte sich hinsichtlich des Alters ein statistisch signifikanter Unterschied zwischen den fünf Gruppen.

In früheren Studien konnte gezeigt werden, dass das Risiko an einer Endometriose zu erkranken nur während des fertilen Alters besteht. Es liegen jedoch unterschiedliche Daten zum Ersterkrankungsalter vor. In älteren Studien ergab sich der Hinweis auf eine zweigipflige Verteilung des altersbezogenen Risikos für Endometriose. Das höchste Risiko wurde mit 44 Lebensjahren angegeben. Eine weitere Altersgruppe mit erhöhtem Risiko lag zwischen 25 und 29 Jahren. In neueren Studien hingegen wurde das höchste Risiko an Endometriose zu erkranken mit 25 bis 29 Jahren angegeben. Das Risiko nahm in allen Studien nach dem 44. Lebensjahr rasch ab. (Houston et al., 1988; Missmer et al., 2004b; Sangi-Haghpeykar und Poindexter, 1995).

Bei unserem Patientenkollektiv lag das mittlere Alter für die operative Erstdiagnose einer Endometriose bei 33,86 Jahren.

All diese Daten bezogen sich jedoch auf die Endometriose insgesamt, es liegen kaum Daten zur Untersuchung des Erkrankungsalters in Abhängigkeit der Art oder Lokalisation der Endometriose vor.

Eine frühere Studie konnte keinen signifikanten Unterschied beim Erkrankungsalter zwischen Patientinnen mit einer tiefinfiltrierenden Endometriose und peritonealer oder ovarieller Endometriose feststellen (Parazzini et al., 2008). Diese Daten konnten wir nicht bestätigen, jedoch hatten wir keinen direkten Vergleich zwischen einer Gruppe mit alleiniger tiefinfiltrierender Endometriose und anderen Gruppen. In einer anderen Studie konnte ein signifikanter Unterschied zwischen Patientinnen mit einer Adenomyose und Endometriose festgestellt werden. Patientinnen mit einer Endometriose waren im Durchschnitt jünger als solche mit einer Adenomyose (Templeman et al., 2008). Diese Daten sind mit unseren Ergebnissen insofern vergleichbar, dass bei unserer Studie ebenfalls eine Gruppe mit einer alleinigen peritonealen Endometriose (Gruppe 1) und eine Gruppe mit alleiniger Adenomyose (Gruppe 3) verglichen wurden. Jedoch vergleicht unsere Studie 
fünf Gruppen miteinander, eine Aussage über einzelne Gruppen ist daher nur der Tendenz nach möglich. Dennoch legen unsere Daten nahe, dass sich Patientengruppen je nach Art und Lokalisation der Endometriose hinsichtlich des Alters unterscheiden, was auf eine unterschiedliche Pathogenese hindeuten könnte.

Als möglicher Bias für dieses Ergebnis ist jedoch die erschwerte Diagnostik einer Adenomyose zu sehen. So kann eine Adenomyose erst bei einer Hysterektomie oder Uterusbiopsie sicher diagnostiziert werden (Reinhold et al., 1996; Ulrich et al., 2013; Walter et al., 2001). Eine Hysterektomie ist bei jungen Patientinnen möglichst zu vermeiden. Dies könnte auch das relativ hohe durchschnittliche Alter bei Erstdiagnose in der Gruppe 3 mit alleiniger Adenomyose erklären.

Es existieren unseres Wissens bislang keine weiteren Daten bezüglich Vergleichen zwischen unterschiedlichen Phänotypen der Endometriose.

In dieser Studie konnte kein signifikanter Zusammenhang zwischen dem Vorstellungsgrund und den fünf Gruppen festgestellt werden. Diese Daten widersprechen den früheren Daten, die Dysmenorrhoe in einem Zusammenhang mit Adenomyose gesehen haben (Bird et al., 1972; Vavilis et al., 1997). Jedoch stehen sie mit den Studien in Einklang, die keinen Zusammenhang zwischen der Ausdehnung der Endometriose und der damit verbundenen Schmerzsymptomatik feststellen konnten (Koninckx et al., 1991). Unsere Daten untersuchten allerdings, ob beim Vergleich verschiedener Endometriose Gruppen ein Zusammenhang mit Schmerzen besteht, nicht ob Schmerzen bei Adenomyose im Vergleich zu einem gesunden Kontrollkollektiv vermehrt auftreten. Zu erwähnen ist, dass sich auch bei uns Patientinnen mit einer alleinigen Adenomyose innerhalb der Gruppe 3 zu 58,3 \% wegen Schmerzen vorgestellt haben. Dies ist die höchste Vorstellungsrate aufgrund von Schmerzen innerhalb einer Gruppe.

In unseren Daten zeigte sich ein signifikanter Zusammenhang zwischen der Schwangerschaftsrate und den fünf untersuchten Gruppen. Daher kann spekuliert werden, dass eine mögliche Infertilität durch Endometriose nicht alle Patientengruppen gleich stark betrifft. Eine Aussage bezüglich Infertilität kann 
mit diesen Daten jedoch nicht gemacht werden, da hier kein Vergleich von Endometriose-Patientinnen mit einem gesunden Kontrollkollektiv vorgenommen worden ist. Dennoch zeigte sich über alle Gruppen hinweg ein hoher Prozentsatz von $62,7 \%$ Nulligravida.

Ein ähnliches Bild zeigte sich bei der Untersuchung der Lebendgeburtenrate. Unsere Daten ergaben hier ebenfalls einen signifikanten Zusammenhang zwischen der Anzahl an Lebendgeburten und den fünf Gruppen. Über alle Gruppen war der Prozentsatz Patientinnen ohne Lebendgeburten mit 72,1\% relativ hoch.

Diese Daten stimmen mit Studien überein, die einen Zusammenhang zwischen niedriger Schwangerschaftsrate und Lebendgeburtenrate und dem Risiko an einer Endometriose zu erkranken nachgewiesen haben (Candiani et al., 1991; Gruppo-Italiano, 1999; Missmer et al., 2004a; Parazzini et al., 1995; Saha et al., 2017).

Interessant ist insbesondere die Gruppe 3 der Patientinnen mit alleiniger Adenomyose. In dieser Gruppe waren mit 42,5\% Nulligravida und 53,6 \% Nullipara die Schwangerschaftsraten und Lebendgeburtenraten deutlich besser als in den anderen Gruppen.

Wie es zu diesem Effekt kommt, bleibt Gegenstand weiterer Untersuchungen. Ein möglicher Einfluss könnte sein, dass das mittlere Alter in dieser Gruppe um 2,04 Jahre über dem durchschnittlichen Erkrankungsalter liegt.

Nimmt man die gefundenen Ergebnisse zusammen, lässt sich darüber spekulieren, ob den unterschiedlichen Ausprägungsformen der Endometriose unterschiedliche Entstehungsmechanismen zugrunden liegen könnten. Ist dies zutreffend, muss dies für eine adäquate Erforschung weiterer Therapieoptionen zukünftig berücksichtigt werden. Wie oben beschrieben, ist allerdings nur wenig über die Pathogenese der Endometriose insgesamt bekannt. Noch weniger ist demnach über die Entstehung einzelner Unterformen bekannt.

Im Folgenden werden die Stärken und Schwächen der vorliegenden Studie kritisch beurteilt. Bei der Untersuchung der Endometriose ist eine Verzerrung der Ergebnisse durch die Selektion des Patientenkollektivs möglich. Die Selektion der Fälle für eine auf Krankenhaus-Fällen basierte Studie ist 
möglicherweise verfälscht durch die medizinische Versorgung, die im Frauenklinikum des Universitätsklinikums Erlangen angeboten wird.

Frauen, die sich wegen Schmerzen in der Frauenklinik Erlangen aufgrund des Behandlungsangebotes bei Verdacht auf Endometriose vorstellen, können die Fallzahl von Patientinnen mit Schmerzen erhöhen. Gleiches gilt für die mögliche vermehrte Vorstellung von Patientinnen mit Infertilität aufgrund der hierfür spezialisierten Einrichtungen. Dies führt zu einer Erhöhung der Fallzahlen in diesen Untergruppen.

Diese Gefahr einer möglichen Verzerrung hinsichtlich der Untergruppen, lässt sich jedoch relativieren, da in der Frauenklinik Erlangen alle gynäkologischen Erkrankungen behandelt werden und es keine ausschließliche Spezialisierung auf Endometriose gibt. Zudem werden in dieser Studie verschiedene FallGruppen miteinander verglichen. Es findet demnach kein Vergleich mit Kontrollgruppen statt. Hierdurch wird das Risiko für einen Bias durch ein selektiertes Patientenkollektiv minimiert und eine Verzerrung durch die oben genannten Punkte ist demnach unwahrscheinlich.

Diese Studie ist ein erster Hinweis darauf, dass bei der zukünftigen Erforschung der Endometriose als Erkrankung eine Differenzierung zwischen verschiedenen Formen der Endometriose vorgenommen werden sollte. Eine genaue Abgrenzung der Endometriosegruppen kann anhand dieser Studie allerdings nicht vorgenommen werden. Um eine zuverlässige Einteilung der Endometriose in Gruppen vornehmen zu können, benötigt es noch weitaus mehr Daten, die genau diese Fragestellung untersuchen. 


\section{Literaturverzeichnis}

Abbas, S., Ihle, P., Koster, I., und Schubert, I. (2012). Prevalence and incidence of diagnosed endometriosis and risk of endometriosis in patients with endometriosisrelated symptoms: findings from a statutory health insurance-based cohort in Germany. European journal of obstetrics, gynecology, and reproductive biology 160, 79-83.

Bird, C.C., McElin, T.W., und Manalo-Estrella, P. (1972). The elusive adenomyosis of the uterus--revisited. American journal of obstetrics and gynecology 112, 583-593.

Blumenkrantz, M.J., Gallagher, N., Bashore, R.A., und Tenckhoff, H. (1981). Retrograde menstruation in women undergoing chronic peritoneal dialysis. Obstetrics and gynecology 57, 667-670.

Burghaus, S., Fasching, P.A., Haberle, L., Rubner, M., Buchner, K., Blum, S., Engel, A., Ekici, A.B., Hartmann, A., Hein, A., et al. (2017). Genetic risk factors for ovarian cancer and their role for endometriosis risk. Gynecologic oncology 145, 142-147.

Burghaus, S., Fehm, T., Fasching, P.A., Blum, S., Renner, S.K., Baier, F., Brodkorb, T., Fahlbusch, C., Findeklee, S., Haberle, L., et al. (2016). The International Endometriosis Evaluation Program (IEEP Study) - A Systematic Study for Physicians, Researchers and Patients. Geburtshilfe und Frauenheilkunde 76, 875-881.

Burghaus, S., Klingsiek, P., Fasching, P.A., Engel, A., Haberle, L., Strissel, P.L., Schmidt, M., Jonas, K., Strehl, J.D., Hartmann, A., et al. (2011). Risk Factors for Endometriosis in a German Case-Control Study. Geburtshilfe und Frauenheilkunde 71, 1073-1079.

Buttram, V.C., Jr. (1979). Cyclic use of combination oral contraceptives and the severity of endometriosis. Fertility and sterility $31,347-348$.

Candiani, G.B., Danesino, V., Gastaldi, A., Parazzini, F., und Ferraroni, M. (1991). Reproductive and menstrual factors and risk of peritoneal and ovarian endometriosis. Fertility and sterility 56, 230-234. 
Cornillie, F.J., Oosterlynck, D., Lauweryns, J.M., und Koninckx, P.R. (1990). Deeply infiltrating pelvic endometriosis: histology and clinical significance. Fertility and sterility 53, 978-983.

Cramer, D.W., und Missmer, S.A. (2002). The epidemiology of endometriosis. Annals of the New York Academy of Sciences 955, 11-22; discussion 34-16, 396-406.

Cramer, D.W., Wilson, E., Stillman, R.J., Berger, M.J., Belisle, S., Schiff, I., Albrecht, B., Gibson, M., Stadel, B.V., und Schoenbaum, S.C. (1986). The relation of endometriosis to menstrual characteristics, smoking, and exercise. Jama 255, 19041908.

Cullen, T. (1908). Adenomyoma of the Uterus. WB Saunders.

Cullen, T. (1920). The distribution of adenomyomas containing uterine mucosa. Arch Surg 1(2), 215-283.

D'Hooghe, T.M., und Debrock, S. (2002). Endometriosis, retrograde menstruation and peritoneal inflammation in women and in baboons. Human reproduction update $8,84-$ 88.

Dmowski, W.P., Steele, R.W., und Baker, G.F. (1981). Deficient cellular immunity in endometriosis. American journal of obstetrics and gynecology 141, 377-383.

Ebert, A.D., Bartley, J., und David, M. (2005). Aromatase inhibitors and cyclooxygenase-2 (COX-2) inhibitors in endometriosis: new questions--old answers? European journal of obstetrics, gynecology, and reproductive biology 122, 144-150.

Eskenazi, B., und Warner, M.L. (1997). Epidemiology of endometriosis. Obstetrics and gynecology clinics of North America 24, 235-258.

Farland, L.V., Eliassen, A.H., Tamimi, R.M., Spiegelman, D., Michels, K.B., und Missmer, S.A. (2017a). History of breast feeding and risk of incident endometriosis: prospective cohort study. BMJ (Clinical research ed) 358, j3778. 
Farland, L.V., Missmer, S.A., Bijon, A., Gusto, G., Gelot, A., Clavel-Chapelon, F., Mesrine, S., Boutron-Ruault, M.C., und Kvaskoff, M. (2017b). Associations among body size across the life course, adult height and endometriosis. Human reproduction (Oxford, England) 32, 1732-1742.

Giudice, L.C. (2003). Genomics' role in understanding the pathogenesis of endometriosis. Seminars in reproductive medicine 21, 119-124.

Gruenwald, P. (1942). Origin of endometriosis from the mesenchyme of the celomic walls. American Journal of Obstetrics \& Gynecology 44, 470-474.

Gruppo-Italiano (1994). Prevalence and anatomical distribution of endometriosis in women with selected gynaecological conditions: results from a multicentric Italian study. Gruppo italiano per lo studio dell'endometriosi. Human reproduction (Oxford, England) 9, 1158-1162.

Gruppo-Italiano (1999). Risk factors for pelvic endometriosis in women with pelvic pain or infertility. Gruppo Italiano per lo Studio dell' endometriosi. European journal of obstetrics, gynecology, and reproductive biology 83, 195-199.

Guo, S.W., und Wang, Y. (2006). The prevalence of endometriosis in women with chronic pelvic pain. Gynecologic and obstetric investigation 62, 121-130.

Hadfield, R.M., Mardon, H.J., Barlow, D.H., und Kennedy, S.H. (1997). Endometriosis in monozygotic twins. Fertility and sterility $68,941-942$.

Heilier, J.F., Donnez, J., Nackers, F., Rousseau, R., Verougstraete, V., Rosenkranz, K., Donnez, O., Grandjean, F., Lison, D., und Tonglet, R. (2007). Environmental and host-associated risk factors in endometriosis and deep endometriotic nodules: a matched case-control study. Environmental research 103, 121-129.

Houston, D.E., Noller, K.L., Melton, L.J., 3rd, und Selwyn, B.J. (1988). The epidemiology of pelvic endometriosis. Clinical obstetrics and gynecology $31,787-800$.

Kennedy, S., Mardon, H., und Barlow, D. (1995). Familial endometriosis. Journal of assisted reproduction and genetics 12, 32-34. 
Kirshon, B., und Poindexter, A.N., 3rd (1988). Contraception: a risk factor for endometriosis. Obstetrics and gynecology 71, 829-831.

Koninckx, P.R., und Martin, D.C. (1992). Deep endometriosis: a consequence of infiltration or retraction or possibly adenomyosis externa? Fertility and sterility $58,924-$ 928.

Koninckx, P.R., Meuleman, C., Demeyere, S., Lesaffre, E., und Cornillie, F.J. (1991). Suggestive evidence that pelvic endometriosis is a progressive disease, whereas deeply infiltrating endometriosis is associated with pelvic pain. Fertility and sterility 55 , 759-765.

Lessey, B.A., Damjanovich, L., Coutifaris, C., Castelbaum, A., Albelda, S.M., und Buck, C.A. (1992). Integrin adhesion molecules in the human endometrium. Correlation with the normal and abnormal menstrual cycle. The Journal of clinical investigation 90, 188195.

Levitan, Z., Eibschitz, I., de Vries, K., Hakim, M., und Sharf, M. (1985). The value of laparoscopy in women with chronic pelvic pain and a "normal pelvis". International journal of gynaecology and obstetrics: the official organ of the International Federation of Gynaecology and Obstetrics 23, 71-74.

Liu, Y., und Zhang, W. (2017). Association between body mass index and endometriosis risk: a meta-analysis. Oncotarget 8, 46928-46936.

Lu, Y., Cuellar-Partida, G., Painter, J.N., Nyholt, D.R., Morris, A.P., Fasching, P.A., Hein, A., Burghaus, S., Beckmann, M.W., Lambrechts, D., et al. (2015). Shared genetics underlying epidemiological association between endometriosis and ovarian cancer. Human molecular genetics 24, 5955-5964.

Mahmood, T.A., und Templeton, A. (1991). Prevalence and genesis of endometriosis. Human reproduction (Oxford, England) 6, 544-549.

Meyer, R. (1919). Über den Stand der Frage der Adenomyosis und Adenomyome im allgemeinen und insbesondere über Adenomyosis seroepithelialis und Adenomyometritis sarcomatosa. Zentralbl Gynäkol 36, 745-750. 
Missmer, S.A., Hankinson, S.E., Spiegelman, D., Barbieri, R.L., Malspeis, S., Willett, W.C., und Hunter, D.J. (2004a). Reproductive history and endometriosis among premenopausal women. Obstetrics and gynecology 104, 965-974.

Missmer, S.A., Hankinson, S.E., Spiegelman, D., Barbieri, R.L., Marshall, L.M., und Hunter, D.J. (2004b). Incidence of laparoscopically confirmed endometriosis by demographic, anthropometric, and lifestyle factors. American journal of epidemiology 160, 784-796.

Moen, M.H. (1994). Endometriosis in monozygotic twins. Acta obstetricia et gynecologica Scandinavica $73,59-62$.

Moen, M.H., und Schei, B. (1997). Epidemiology of endometriosis in a Norwegian county. Acta obstetricia et gynecologica Scandinavica 76, 559-562.

Neme, R.M., Andrade, D.C., Brescia, M., Petta, C.A., Ferriani, R., und Abrao, M.S. (2002). Epidemiological study on risk factors of pelvic endometriosis in Brazil. Fertility and sterility 77, S37.

Nezhat, F., Datta, M.S., Hanson, V., Pejovic, T., Nezhat, C., und Nezhat, C. (2008). The relationship of endometriosis and ovarian malignancy: a review. Fertility and sterility $90,1559-1570$.

Nisolle, M., und Donnez, J. (1997). Peritoneal endometriosis, ovarian endometriosis, and adenomyotic nodules of the rectovaginal septum are three different entities. Fertility and sterility 68, 585-596.

Oosterlynck, D.J., Cornillie, F.J., Waer, M., Vandeputte, M., und Koninckx, P.R. (1991). Women with endometriosis show a defect in natural killer activity resulting in a decreased cytotoxicity to autologous endometrium. Fertility and sterility $56,45-51$.

Parazzini, F., Cipriani, S., Bianchi, S., Gotsch, F., Zanconato, G., und Fedele, L. (2008). Risk factors for deep endometriosis: a comparison with pelvic and ovarian endometriosis. Fertility and sterility 90, 174-179. 
Parazzini, F., Ferraroni, M., Fedele, L., Bocciolone, L., Rubessa, S., und Riccardi, A. (1995). Pelvic endometriosis: reproductive and menstrual risk factors at different stages in Lombardy, northern Italy. Journal of epidemiology and community health 49, 61-64.

Parazzini, F., La Vecchia, C., Franceschi, S., Negri, E., und Cecchetti, G. (1989). Risk factors for endometrioid, mucinous and serous benign ovarian cysts. International journal of epidemiology $18,108-112$.

Pearce, C.L., Templeman, C., Rossing, M.A., Lee, A., Near, A.M., Webb, P.M., Nagle, C.M., Doherty, J.A., Cushing-Haugen, K.L., Wicklund, K.G., et al. (2012). Association between endometriosis and risk of histological subtypes of ovarian cancer: a pooled analysis of case-control studies. The Lancet Oncology 13, 385-394.

Pinsonneault, O., und Goldstein, D.P. (1985). Obstructing malformations of the uterus and vagina. Fertility and sterility 44, 241-247.

Reinhold, C., McCarthy, S., Bret, P.M., Mehio, A., Atri, M., Zakarian, R., Glaude, Y., Liang, L., und Seymour, R.J. (1996). Diffuse adenomyosis: comparison of endovaginal US and MR imaging with histopathologic correlation. Radiology 199, 151-158.

Renner, S.P., Boosz, A.S., Burghaus, S., Maihofner, C., Beckmann, M.W., Fasching, P.A., und Jud, S.M. (2012). Visual pain mapping in endometriosis. Archives of gynecology and obstetrics 286, 687-693.

Renner, S.P., Rix, S., Boosz, A., Lermann, J.H., Strissel, P.L., Thiel, F.C., Oppelt, P., Beckmann, M.W., und Fasching, P.A. (2010). Preoperative pain and recurrence risk in patients with peritoneal endometriosis. Gynecological endocrinology : the official journal of the International Society of Gynecological Endocrinology 26, 230-235.

Renner, S.P., Strick, R., Oppelt, P., Fasching, P.A., Engel, S., Baumann, R., Beckmann, M.W., und Strissel, P.L. (2006). Evaluation of clinical parameters and estrogen receptor alpha gene polymorphisms for patients with endometriosis. Reproduction (Cambridge, England) 131, 153-161. 
Rokitansky, C. (1860). Über Uterusdrüsen-Neubildung in Uterus- und OvarialSarcomen. (On the neoplasm of uterus glands on uterine and ovarian sarcomas). Zeitschr Ges Aerzte Wien 16, 577-581.

Saha, R., Kuja-Halkola, R., Tornvall, P., und Marions, L. (2017). Reproductive and Lifestyle Factors Associated with Endometriosis in a Large Cross-Sectional Population Sample. Journal of women's health (2002) 26, 152-158.

Sampson, J. (1921). Perforating hemorrhagic (chocolate) cysts of the ovary, their importance and especially their relation to pelvic adenomas of endometrial type ("Adenomyoma" of the uterus, rectovaginal septum, sigmoid, etc.). Arch Surg 3(2), 245-323.

Sampson, J. (1927a). Peritoneal endometriosis due to the menstrual dissemination of endometrial tissue into the peritoneal cavity. American journal of obstetrics and gynecology $14,422-469$.

Sampson, J.A. (1927b). Metastatic or Embolic Endometriosis, due to the Menstrual Dissemination of Endometrial Tissue into the Venous Circulation. The American journal of pathology 3, 93-110.143.

Sangi-Haghpeykar, H., und Poindexter, A.N., 3rd (1995). Epidemiology of endometriosis among parous women. Obstetrics and gynecology 85, 983-992.

Saraswat, L., Ayansina, D.T., Cooper, K.G., Bhattacharya, S., Miligkos, D., Horne, A.W., und Bhattacharya, S. (2017). Pregnancy outcomes in women with endometriosis: a national record linkage study. BJOG : an international journal of obstetrics and gynaecology $124,444-452$.

Signorello, L.B., Harlow, B.L., Cramer, D.W., Spiegelman, D., und Hill, J.A. (1997). Epidemiologic determinants of endometriosis: a hospital-based case-control study. Annals of epidemiology 7, 267-741.

Simpson, J.L., und Bischoff, F. (2003). Heritability and candidate genes for endometriosis. Reproductive biomedicine online 7, 162-169. 
Simpson, J.L., und Bischoff, F.Z. (2002). Heritability and molecular genetic studies of endometriosis. Annals of the New York Academy of Sciences 955, 239-251; discussion 293-235, 396-406.

Simpson, J.L., Elias, S., Malinak, L.R., und Buttram, V.C., Jr. (1980). Heritable aspects of endometriosis. I. Genetic studies. American journal of obstetrics and gynecology 137, 327-331.

Stefansson, H., Geirsson, R.T., Steinthorsdottir, V., Jonsson, H., Manolescu, A., Kong, A., Ingadottir, G., Gulcher, J., und Stefansson, K. (2002). Genetic factors contribute to the risk of developing endometriosis. Human reproduction (Oxford, England) 17, 555559.

Takayama, K., Zeitoun, K., Gunby, R.T., Sasano, H., Carr, B.R., und Bulun, S.E. (1998). Treatment of severe postmenopausal endometriosis with an aromatase inhibitor. Fertility and sterility 69, 709-713.

Templeman, C., Marshall, S.F., Ursin, G., Horn-Ross, P.L., Clarke, C.A., Allen, M., Deapen, D., Ziogas, A., Reynolds, P., Cress, R., et al. (2008). Adenomyosis and endometriosis in the California Teachers Study. Fertility and sterility 90,415-424.

Treloar, S.A., Bell, T.A., Nagle, C.M., Purdie, D.M., und Green, A.C. (2010). Early menstrual characteristics associated with subsequent diagnosis of endometriosis. American journal of obstetrics and gynecology 202, 534.e531-536.

Ulrich, U., Buchweitz, O., Greb, R., Keckstein, J., von Leffern, I., Oppelt, P., Renner, S.P., Sillem, M., Stummvoll, W., und Schweppe, K.W. (2013). Interdisciplinary S2k Guidelines for the Diagnosis and Treatment of Endometriosis: Short Version - AWMF Registry No. 015-045, August 2013. Geburtshilfe und Frauenheilkunde 73, 890-898.

Valle, R.F., und Sciarra, J.J. (2003). Endometriosis: treatment strategies. Annals of the New York Academy of Sciences 997, 229-239.

Vavilis, D., Agorastos, T., Tzafetas, J., Loufopoulos, A., Vakiani, M., Constantinidis, T., Patsiaoura, K., und Bontis, J. (1997). Adenomyosis at hysterectomy: prevalence and 
relationship to operative findings and reproductive and menstrual factors. Clinical and experimental obstetrics \& gynecology $24,36-38$.

Vercellini, P., Frontino, G., De Giorgi, O., Pietropaolo, G., Pasin, R., und Crosignani, P.G. (2003). Continuous use of an oral contraceptive for endometriosis-associated recurrent dysmenorrhea that does not respond to a cyclic pill regimen. Fertility and sterility $80,560-563$.

Vercellini, P., Frontino, G., Pietropaolo, G., Gattei, U., Daguati, R., und Crosignani, P.G. (2004). Deep endometriosis: definition, pathogenesis, and clinical management. The Journal of the American Association of Gynecologic Laparoscopists 11, 153-161.

Vigano, P., Parazzini, F., Somigliana, E., und Vercellini, P. (2004). Endometriosis: epidemiology and aetiological factors. Best practice \& research Clinical obstetrics \& gynaecology 18, 177-200.

Vitonis, A.F., Hankinson, S.E., Hornstein, M.D., und Missmer, S.A. (2010). Adult physical activity and endometriosis risk. Epidemiology (Cambridge, Mass) 21, 16-23.

Walter, A.J., Hentz, J.G., Magtibay, P.M., Cornella, J.L., und Magrina, J.F. (2001). Endometriosis: correlation between histologic and visual findings at laparoscopy. American journal of obstetrics and gynecology 184, 1407-1411; discussion 1411-1403.

Weiss, G., Maseelall, P., Schott, L.L., Brockwell, S.E., Schocken, M., und Johnston, J.M. (2009). Adenomyosis a variant, not a disease? Evidence from hysterectomized menopausal women in the Study of Women's Health Across the Nation (SWAN). Fertility and sterility 91, 201-206.

Yu, H.C., Lin, C.Y., Chang, W.C., Shen, B.J., Chang, W.P., und Chuang, C.M. (2015). Increased association between endometriosis and endometrial cancer: a nationwide population-based retrospective cohort study. International journal of gynecological cancer : official journal of the International Gynecological Cancer Society 25, 447-452.

Zanetta, G.M., Webb, M.J., Li, H., und Keeney, G.L. (2000). Hyperestrogenism: a relevant risk factor for the development of cancer from endometriosis. Gynecologic oncology $79,18-22$. 
Abkürzungsverzeichnis

$\begin{array}{ll}\text { ANOVA } & \text { Analysis of Variances } \\ \text { BMI } & \text { Body Mass Index } \\ \text { CPP } & \text { chronic pelvic pain } \\ \text { eCRF } & \text { elektronisches Case-Report-Form } \\ \text { IEEP } & \text { International Endometriosis Evaluation Program } \\ \text { SEF } & \text { Stiftung Endometriose-Forschung } \\ \text { TIE } & \text { tiefinfiltrierende Endometriose } \\ \text { usw. } & \text { und so weiter }\end{array}$


Anhang 


\section{Anlage 1: Übersicht "Medical history"}

\begin{tabular}{l}
$\begin{array}{l}\text { Examination plan } \quad \text { Registration } \\
\text { Planned visits } \\
\text { Data entry }\end{array}$ \\
0 Basis data [E-CERT] \\
1 Medical history [E-CERT] \\
2 Lifestyle and reproduction \\
3 Laboratory \\
4 Endometriosis: symptoms and diagnostics [E-CERT] \\
\hline Endometriosis: therapy [E-CERT] \\
6 Follow up [E-CERT]
\end{tabular}

1.1 Disease history [E-CERT] 1.2 Surgery history [E-CERT]


Anlage 2: Übersicht "Endometriosis therapy"

\begin{tabular}{l} 
Examination plan Registration \\
$\begin{array}{l}\text { Planned visits } \\
\text { Data entry }\end{array}$ \\
0 Basis data [E-CERT] \\
1 Medical history [E-CERT] \\
2 Lifestyle and reproduction \\
3 Laboratory \\
4 Endometriosis: symptoms and diagnostics [E-CERT] \\
\hline Endometriosis: therapy [E-CERT] \\
6 Follow up [E-CERT]
\end{tabular}




\section{Anlage 3: Formular "Surgery history"}

\subsection{Surgery history}

1.2.1 General surgery history

NOTE: These questions refer to the past respectively the time between the visits

a. Did the patient have a hysterectomy (meanwhile)?

$\odot$ No $\bigcirc$ Yes $\bigcirc$ Unknown $\bigcirc$ NA

b. Did the patient have an ovarectomy (meanwhile)?

$\odot \bigcirc$ No $\bigcirc$ Yes $\bigcirc$ Unknown $\bigcirc$ NA

c. Did the patient have a tubal ligation or salpingektomie (meanwhile)?

$\odot \bigcirc$ No $\bigcirc$ Yes $\bigcirc$ Unknown $\bigcirc$ NA

d. Did the patient have another abdominal surgery (meanwhile)?

$\odot \bigcirc$ No $\bigcirc$ Yes $\bigcirc$ Unknown $\bigcirc$ NA

1.2.2 Endometriosis surgery history

a. Did the patient have any surgery because of endometriosis or with diagnosis of endometriosis in the past / since the last visit?

$\odot \bigcirc$ No $\bigcirc$ Yes $\bigcirc$ Unknown $\bigcirc \mathrm{NA}$

$\begin{array}{ll}\text { Last saved by } & \text { Reason } \\ \text { at } & \text { Project version } \ldots\end{array}$

\section{Anlage 4: Formular "Surgery therapy"}

\subsection{Surgery therapy [E-CERT]}

Did the patient have a surgery because of suspected endometriosis in the current visit or is there an incidental finding of endometriosis?

Please document surgeries before the first visit or between the visits in the form "Medical History/Surgery History".

Please just document the current surgery.

$\odot$ No OYes OUnknown ONA

$\begin{array}{ll}\text { Last saved by } & \text { Reason } \\ \text { at } & \text { Project version }\end{array}$




\section{Anlage 5: Subformular "Surgical therapy"}

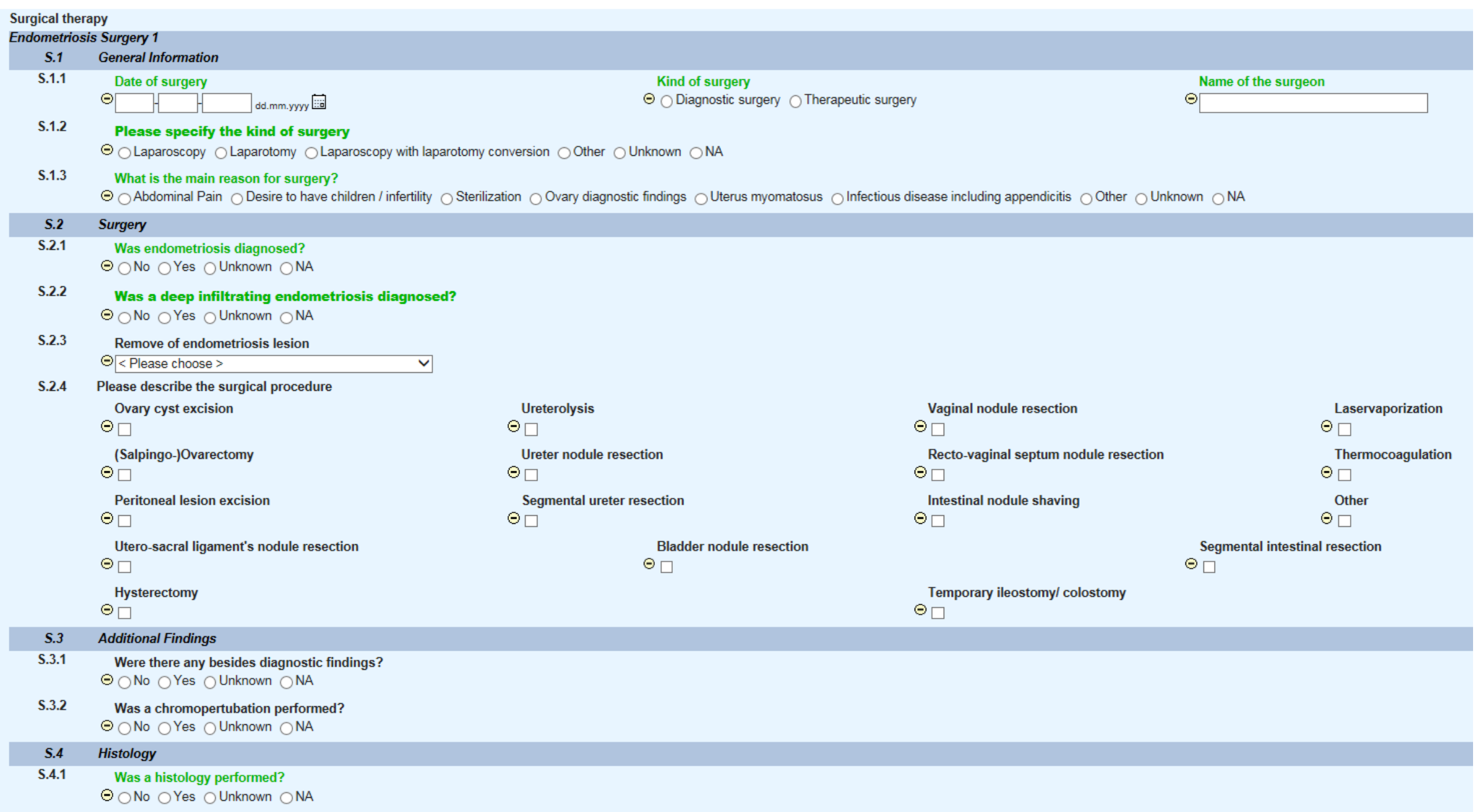




\section{Anlage 6: Übersicht "Basis data"}

Examination plan Registration
$\begin{aligned} & \text { Planned visits } \\ & \text { Data entry }\end{aligned}$
0 Basis data [E-CERT]
1 Medical history [E-CERT]
2 Lifestyle and reproduction
3 Laboratory




\section{Anlage 7: Formular "Registration"}

0.10 Registration [E-CERT]

0.1.1 Date of birth

$\ominus \square-\square$ dd.mm.yyyy 圆

0.1.2 Is the patient willing to participate as part of a population-based control group? $\ominus \bigcirc$ Yes $\bigcirc$ No $\bigcirc$ Unknown

0.1.3 Date of informed consent / study entry $\ominus \square$ dd.mm.yyyy 圆

0.1.4 Date the patient's information (epidemiological questionnaire/ interview and medical history) was obtained. (This date is reffered to as "reference date" in the following questions.).

$\odot \square$ dd.mm.yyyy 圆

PatID1-Endometriosis Center (KIS-No) $\Theta$

\begin{tabular}{ll}
\hline Last saved by & Reason \\
at & Project version .-
\end{tabular}

\section{Anlage 8: Formular "Entry specifications"}
0.2 Entry specifications [E-CERT]
0.2.1 Setting
The patient is ...
$\odot$ outpatient $\bigcirc$ inpatient $\bigcirc$ out- and inpatient $\bigcirc$ external $\bigcirc$ Unknown
Is there a surgery planned in this visit?
$\ominus \bigcirc$ No $\bigcirc$ Yes $\bigcirc$ Unknown $\bigcirc \mathrm{NA}$

0.2.2 What is the MAIN reason for consultation?

Registration as type of case

$\Theta \bigcirc$ Women with abdominal / pelvic pain

Women with sub- and infertility

Women with other reason(s) for referral/ admittance/ surgery 


\section{Anlage 9: Übersicht "Lifestyle und reproduction"}

\begin{tabular}{l}
\hline Examination plan Registration \\
$\begin{array}{l}\text { Planned visits } \\
\text { Data entry }\end{array}$ \\
0 Basis data [E-CERT] \\
1 Medical history [E-CERT] \\
2 Lifestyle and reproduction
\end{tabular}

$\odot \sqcup \quad \odot \square \quad \odot \square \quad \odot \square$

2.1 Lifestyle $\quad 2.2$ Reproduction characteristics $\quad 2.3$ Artificial reproduction $\quad 2.4$ Pregnancies 


\section{Anlage 10: Formular "Pregnancies"}

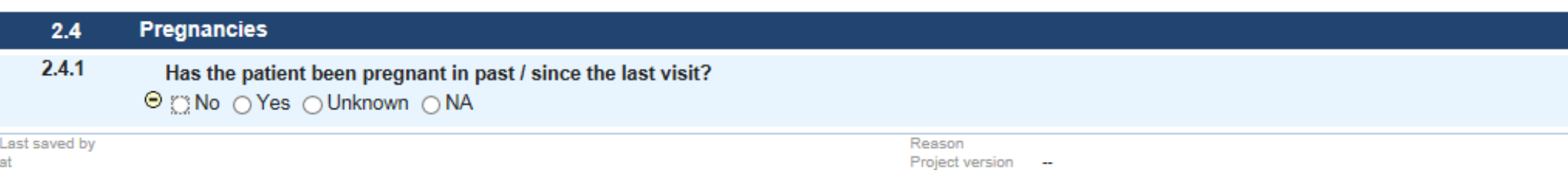

\section{Anlage 11: Subformular "Pregnancy 1"}

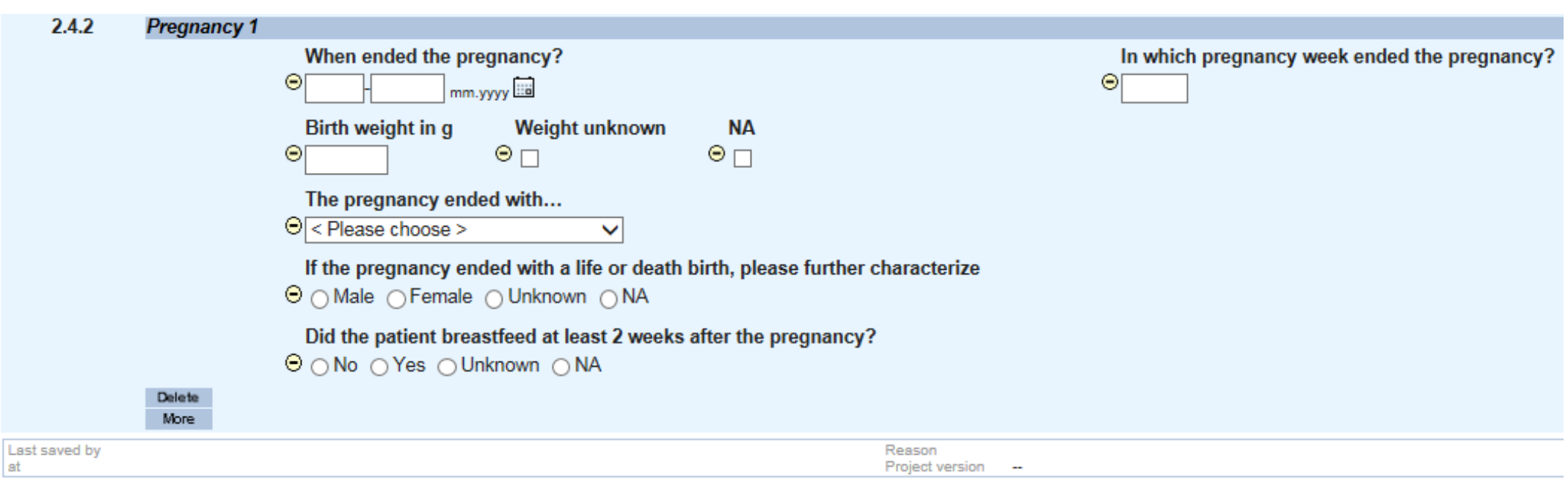




\section{Anlage 12: Formular "Lifestyle"}

$2.1 \quad$ Lifestyle

2.1.1 General nutrition

a. How often does the patient eat fresh fruits and vegetables?

$\odot$ Never $\bigcirc 1-2$ times per month $\bigcirc 1-2$ times per week $\bigcirc$ Nearly daily/daily $\bigcirc$ Unknown $\bigcirc$ NA

b. How often does the patient eat pork or beef (red meat)?

$\ominus \bigcirc$ Never $\bigcirc 1-2$ times per month $\bigcirc 1-2$ times per week $\bigcirc$ Nearly daily / daily $\bigcirc$ Unknown $\bigcirc$ NA

c. How often does the patient eat poultry or fish (white meat)?

$\ominus \bigcirc$ Never $\bigcirc 1-2$ times per month $\bigcirc 1-2$ times per week $\bigcirc$ Nearly daily / daily $\bigcirc$ Unknown $\bigcirc N A$

d. Are there any digestion problems known? $\ominus \bigcirc$ No $\bigcirc$ Yes $\bigcirc$ Unknown $\bigcirc$ NA

\subsection{2 $B M$}

a. What is the current weight in $\mathrm{kg}$ ? $\odot$

b.
Weight unknown

$\ominus \square$

Height unknown
$\ominus \square$
What is the current height in $\mathrm{cm}$ ? $\ominus$ 


\section{Anlage 13: Formular "Reproductive CHaracteristics"}

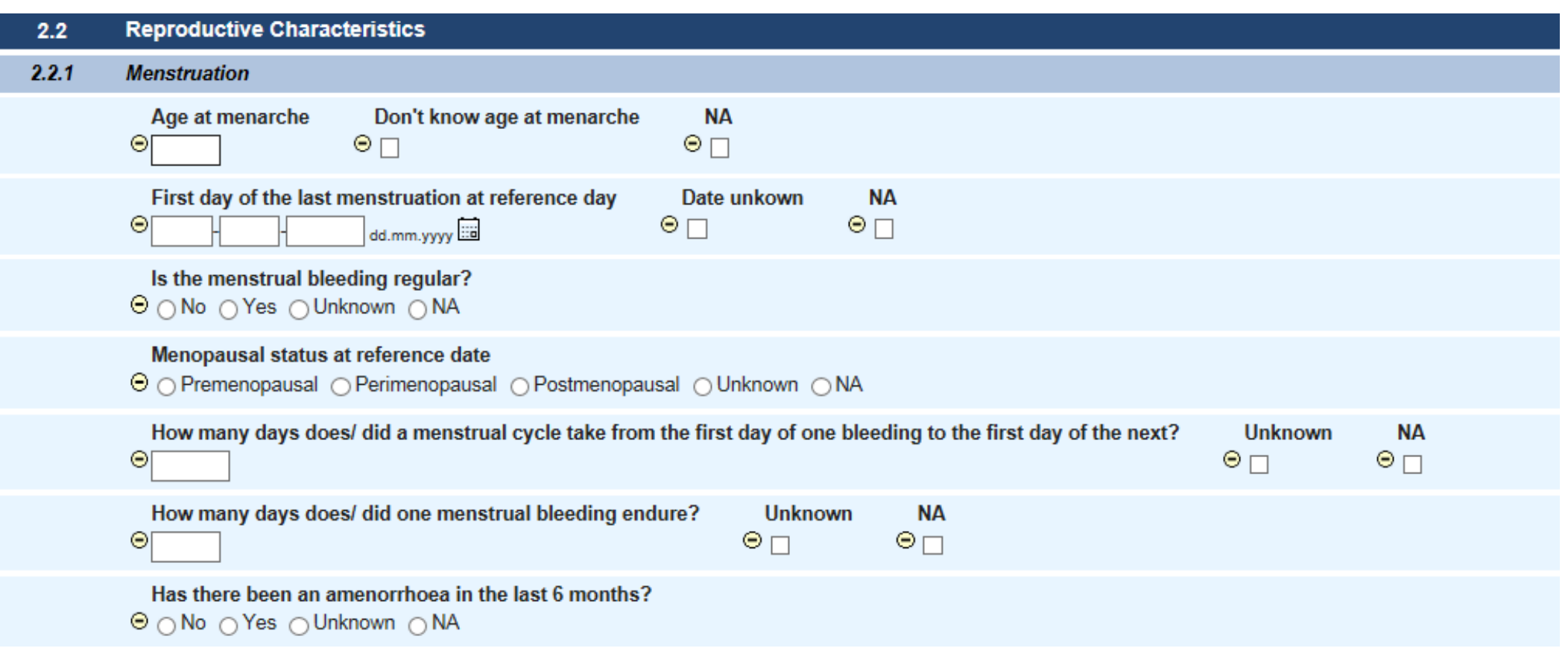




\section{Anlage 14: Formular "Basic patient characteristics"}

0.3 Basic patient characteristics

0.3.1 Ethnicity

a. Please specify the ethnic origin of patient

$\Theta<$ Please choose $>$

0.3.2 Socio-economic status

a. Specify the highest level of education

$\Theta<$ Please choose > V

b. Current employment

$\Theta<$ Please choose $>\checkmark$

0.3.3 Marital status

Specify the current marital status

$\ominus<$ Please choose >

Last saved by Reason




\section{Danksagung}

Mein besonderer Dank gilt zunächst allen Studienteilnehmerinnen. Ohne sie wäre diese Arbeit nicht möglich gewesen. Generell ermöglicht erst ihre Teilnahme an solchen Forschungsprojekten den wissenschaftlichen Fortschritt.

Zudem gilt mein besonders herzlicher Dank Prof. Dr. med. Peter Fasching. Er stand mir stets mit Rat und Tat zur Seite und unterstützte mich damit uneingeschränkt bei der Fertigstellung der Arbeit.

Ihm danke ich für seine Unterstützung während des gesamten Promotionsverfahrens. Ohne dessen konstruktive Kritik hätte diese Arbeit nicht ihre endgültige Form gefunden.

Zudem möchte ich mich bei Prof. Dr. med. Matthias W. Beckmann, Direktor der Frauenklinik der Universität Erlangen für die Überlassung des Themas und die Möglichkeit, diese Arbeit an seinem Institut durchzuführen, bedanken. Seine investierte Zeit und Kritik brachte diese Arbeit maßgeblich voran.

Außerdem möchte ich der gesamten Arbeitsgruppe für die erfolgreiche $\mathrm{Zu}$ sammenarbeit danken.

Abschließend möchte ich mich von Herzen bei meiner Familie für deren vielseitige Unterstützung, Geduld und Motivation während der gesamten Promotionszeit bedanken. 